\title{
Cramer-Rao bounds in the estimation of time of arrival in fading channels
}

\author{
René Játiva ${ }^{1 *}$ (10) and Josep Vidal ${ }^{2}$
}

\begin{abstract}
This paper computes the Cramer-Rao bounds for the time of arrival estimation in a multipath Rice and Rayleigh fading scenario, conditioned to the previous estimation of a set of propagation channels, since these channel estimates (correlation between received signal and the pilot sequence) are sufficient statistics in the estimation of delays. Furthermore, channel estimation is a constitutive block in receivers, so we can take advantage of this information to improve timing estimation by using time and space diversity. The received signal is modeled as coming from a scattering environment that disperses the signal both in space and time. Spatial scattering is modeled with a Gaussian distribution and temporal dispersion as an exponential random variable. The impact of the sampling rate, the roll-off factor, the spatial and temporal correlation among channel estimates, the number of channel estimates, and the use of multiple sensors in the antenna at the receiver is studied and related to the mobile subscriber positioning issue. To our knowledge, this model is the only one of its kind as a result of the relationship between the space-time diversity and the accuracy of the timing estimation.
\end{abstract}

Keywords: Cramer-Rao bounds, CRB, Time of arrival, TOA, Mobile subscriber location

\section{Introduction}

Positioning of a mobile subscriber is a complex task that has the capability of adding value to services and applications such as navigational aids, and patient and personnel monitoring [1]. It is also useful when performing driving tests [2] and helps to enhance the mobile network allocation resources, handover decisions, etc. [3]. Permanent research is being developed in this area with increasing complexity $[4,5]$ to sustain in time the adaptation of these principles to new emerging technologies $[6,7]$.

Network-based positioning is performed through the estimation of signal parameters involved in the communication process. These parameters may include time of arrival (TOA), direction of arrival (DOA), observed time differences of arrival (OTDOA), signal strength (SS), etc. Estimators based on time are preferred over those based on bearing due to their better resolution, but hybrid techniques may also be implemented to reduce positioning variance error [8]. Furthermore, SS measurements may be added to TOA- or OTDOA-based methods to increase

*Correspondence: rjativa@usfq.edu.ec

'Universidad San Francisco de Quito, Diego de Robles y Pampite, Quito, Ecuador

Full list of author information is available at the end of the article positioning accuracy in line of sight (LOS) environments, such as indoors, using ultra-wide band radios (UWB) or wireless sensor networks [9-12]. The position may be estimated from these parameters using propagation relations or pattern recognition techniques $[9,13]$.

Permanent efforts have been made to characterize wireless channels [14-17], and practical estimators have been derived. For instance, Bengtsson [18], Besson [19], and Valaee [20] have described several techniques based on signal subspace to estimate DOA and angular spread for wireless dispersed signal, whereas Raleigh [21] and Wax [22], among others [23] have studied the problem of Joint Space-Time Estimation in a multipath environment.

However, since the final performance of a specific positioning technique depends on the way signal parameters are estimated, a general comparison of the different techniques is difficult. For this reason, we study the problem of TOA estimation in both Rice and Rayleigh propagation conditions from a Cramer-Rao perspective since the lower bound of an unbiased estimator determines the best possible behavior in the estimation of a particular parameter of interest. In this way, the limiting variances for timing can be used to get an insight about the positioning accuracy. 
Other bounds besides the Cramer-Rao bound (CRB) exist, such as the Barankin bound (BB) [24] or the ZivZakai bound (ZZB) [25]. The BB claims to be the greatest lower bound on Mean Squares Error (MSE) for a uniformly unbiased estimator, but it is generally incomputable analytically [24]; the ZZB is useful in environments such as GNSS (Global Navigation Satellite System), where the signal-to-noise ratio (SNR) is very low and the CRB cannot be used. However, we prefer the use of the CRB since it is adequate for modeling Gaussian processes $[25,26]$. In addition, it is useful to identifying if a particular estimator is the minimum variance unbiased (MVU) estimator and if a MVU estimator really exists. Furthermore, in the case that such a MVU estimator does not exist, it can still predict the performance of maximum likelihood estimates in an approximate sense for certain conditions of high (SNR) or when a large number of observations is available [26].

In addition to the deterministic CRB which models some parameters as unknown deterministic variables as in our case, the Bayesian CRB (BCRB) models some unknown parameters as random. However, it has been reported that in certain cases, results predicted by CRBs or BCRBs are too optimistic and some modifications to the classical CRBs have been proposed lately. This requires the postponement of the application of an expectation operator required for Fisher information matrix (FIM) computation, in a way that matrix inversion is performed first and then as a second step, an expectation operator is applied to compute the modified CRB (MCRB) [27]. These latter variations of the CRBs are out of the scope of this paper.

Although other approaches exist in the computation of the CRB for TOA, to the best of our knowledge, our model is in fact the most complete of its kind in the literature, since it incorporates a way to take into account spatial and temporal correlation among channel estimates, and the impact of the roll-off factor, in addition to the number of sensors and the number of estimates that are typical from other approaches [28-32]. Our model also assumes an exponential dispersion for delays, which is characteristic of mobile channels, instead of just a few paths [28-31, 33, 34]. Furthermore, we provide asymptotic expressions for the general case, suitable for high levels of SNR and a large number of channel vector estimates $[35,36]$.

Finally, it is important to point out that our model assumes no biased measurements; in other words, we assume that the first arrival, although weak due to the shadowing (non LOS condition), is in fact related to the LOS component. In fact, the non LOS (NLOS) condition is an important issue for the location problem and therefore its identification and mitigation are still a current research topic [37-46]. For example, it is conceptually interesting to consider the use of Bayesian mechanisms which take advantage of system dynamics and add any previous knowledge available, in order to smartly select, among a set of measurements, those with the capacity to lead to a more confident estimation. Some of these strategies use variations of the Kalman filter (KF) to incorporate this intelligence into the Positioning Computing Function [42-44] and employ some lateral information such as the signal quality indicator associated with LOS/NLOS in [42] or prior knowledge to adjust NLOS data toward the corresponding LOS values [40].

The structure of the paper is as follows. Section 2 first introduces the assumptions on the signal model and presents a brief discussion related to signal dispersion and the coherence time for delays, which is required prior to introducing the channel model, and lastly the procedures to compute the true CRBs as well as the asymptotic expressions for the timing. Section 3 presents the CRBs characteristics for LOS and NLOS models and contrasts these results with those provided by a practical timing estimator. Section 4 summarizes the main observations, conclusions and recommendations.

\section{Signal model}

\subsection{Model assumptions}

The following assumptions are taken into consideration:

AS 1. Channel introduces multipath propagation; therefore, the signal is dispersed in space and time from the LOS component. Statistical independence for angular and temporal dispersion processes is assumed. Independence is a reasonable assumption because each path is affected in a different and unpredictable way by the propagation environment. The first TOA is the parameter of interest for the problem, while the angular parameters are nuisance parameters required for the characterization of the CRB.

AS 2. Despite the channel having a coherence time for the taps amplitudes [47, 48], delay and even angle information may remain within tolerable limits much longer due to the high proportion between light speed and mobile speed, and the relatively great distances between transmitter and receiver. Therefore, many channel estimates can be collected in time so as to improve the accuracy of the timing and angle estimates [49].

AS 3. The first arrival is analyzed as the one bearing timing for position information. Measures for TOA are computed from channel estimates available at the receiver through a correlation function. A full maximum likelihood (ML) estimation of all propagation parameters (delays and angles) is considered an approach that is too expensive in a dispersive channel, where the number of parameters could be too large, and might lead to 
inconsistent estimates if the available number of channels is low.

AS 4. Noise present in the channel estimates is white and Gaussian, which is a reasonable assumption after the matched filter [50]. Our analysis does not strictly consider a multi-user environment, but this assumption is reasonable even in this case since all other users have been at least partially canceled.

AS 5. The power angular spectrum (PAS) is symmetric and dispersive and exhibits just a single mode with the mean value associated to the right angular position of the transmitter (the UE if the PAS is computed at the base station (BS)). Gaussian and Laplacian [15, 51-54] models usually describe the marginal probability $P(\theta)$ in (1):

$$
P(\theta)=\int P(\theta, \tau) d \tau
$$

A single modality is a reasonable assumption as long as the channel bandwidth is large, and therefore the channel estimated at chip time includes only rays impinging from a narrow solid angle. Furthermore, some experimental evidence shows that the probability of having more than a cluster in a typical urban environment reaches $13 \%$ and it reduces to $8 \%$ in suburban areas [15].

AS 6. A continuous power spectrum is used in delay for the marginal function $P(\tau)$ in $(2)$ :

$$
P(\tau)=\int P(\theta, \tau) d \theta
$$

It is assumed to fit an exponential shaping [47], estimated at a fraction of the chip time. For the extraction of timing information, the same angular distribution for all delays is assumed. This may not be very realistic, but it allows for reducing the number of parameters in the model and keeps the problem tractable $[34,54]$.

Note that our basic model may be used to study some NLOS scenarios since each tap of the channel impulse response is a zero mean random variable. Certainty, an LOS situation implies a non-zero mean where the first arrival is considered as the one conveying unbiased location of the UE. Therefore, and in order to achieve more general results, this basic model has been enhanced to introduce the LOS condition as a symmetric kernel for the angular distribution with a peak discontinuity at the true angular position of the source, as it is described in Section 2.4.2.

AS 7. A first-order autoregressive (AR) Markov process for the evolution of the channel along the time due to Doppler is assumed [55].

This model is very convenient for the purpose of location, since only a few parameters of interest are going to be computed, rather than all delays and angles which are usually nuisance parameters. First arrival is the desired parameter, since in most cases, positioning accuracy just slightly improves with the use of the whole multipath coming from the LOS nodes in comparison with the use of their first components only [33, 56]. Furthermore, in a practical positioning system deployment, the transmission of these parameters to a remote device is required [57], so a lower number of parameters reduces the signaling channel bandwidth.

\subsection{Coherence time and delay dispersion}

Channel estimation is limited by mobility. Coherence time corresponds to the interval in which the channel is essentially considered time invariant, and it is related to the inverse of the Doppler variation. It is easy to perceive that timing estimation will be affected at least by an error related to the displacement of the MS (mobile station) in the observation interval. Hence, this coherence time for the first arriving signal may be related with the maximum allowed delay uncertainty $(\eta)$ introduced by the movement and the radial component of the speed vector $\left(v_{r}\right)$ of the mobile, as it was commented in [35]. When $v_{r}$ is very small, errors due to displacements are also small and the number of available channel estimates required to keep a specified uncertainty grows, and hence a practical limit has to be imposed to the observation time $T_{a c q}$ (associated with the latency experienced by the user in the availability of the position) in order to deliver the timing to the Position Computing Function (PCF). Therefore, the number of channel estimates reaches a finite limit.

Figure 1 exhibits a set of characteristics related to the mean number of channel vector estimates $(K)$, the mobile speed and the expected accuracy in TOA estimation for typical parameters of a Wideband Code Division Multiple Access (WCDMA) system, a chip rate of 3.84 Mcps and a timeslot of $666.66 \mu \mathrm{s}[35,36]$. Note that the maximum number of observations is limited by the acquisition time and the mobile speed. A faster MS will have less time for channel acquisition, and a compromise will be required to achieve the best timing accuracy.

From a systemic viewpoint, mobile location may be improved when system dynamics [49] and previous knowledge from the statistics of the measures are used [42] and also from the use of a heterogeneous set of measurements $[12,44,49]$.

\subsection{Signal model}

The observed signal is a set of $K$ channel vector estimates collected over a set of $N_{s}$ sensors. Each channel vector estimate $\mathbf{z}$ is of length $N$, and it is estimated from correlation of a known sequence with the received signal. Notations of frequent use within this paper are summarized in Table 1. 


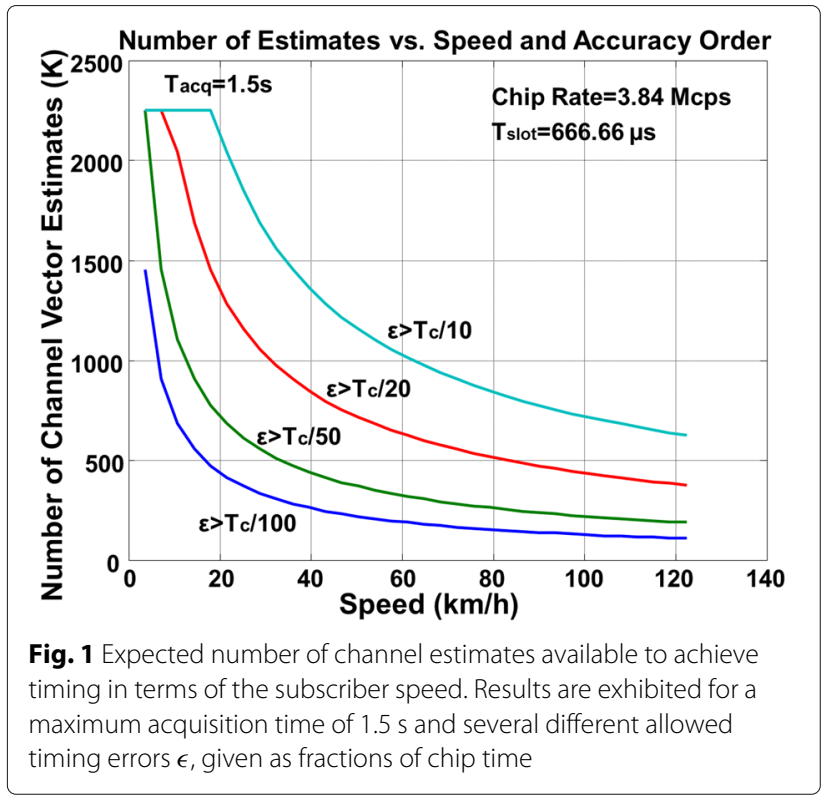

The signal received by $j$-th sensor is noted as $y^{(j)}(\mathrm{t})$ and is expressed in (3) as the summation of multipath components and noise $n^{(j)}(\mathrm{t})$. Each replica of the transmitted signal $x(t)$ arriving at delay $\tau_{i}$ is affected by (i) a timevarying unit-power steering coefficient $b_{i j}(t)$, associated with the path impinging angle in relation to the antenna array geometry; (ii) the path attenuation factor $\gamma_{i}(t)$; and (iii) a time invariant (over time intervals of length $K T_{s}$ ) Doppler frequency $f_{i}$, where $T_{s}$ is the time between two

Table 1 Notations

\begin{tabular}{|c|c|}
\hline Notation & Description \\
\hline$N, K, N_{S}$ & $\begin{array}{l}\text { Respectively, the number of lags at the observation } \\
\text { windows, the number of channel vector estimates, } \\
\text { and the number of sensors at the antenna array }\end{array}$ \\
\hline $\mathbf{z} \in C^{N K N_{5} \times 1}$ & Vector containing the channel estimates \\
\hline $\mathbf{w} \in C^{N K N_{5} \times 1}$ & Vector containing the estimation noise \\
\hline $\mathbf{R}_{\mathbf{z}}$ & Correlation matrix for channel estimates \\
\hline $\mathbf{R}_{\phi}(\rho)$ & Spatial correlation matrix (spatial correlation vector) \\
\hline $\mathbf{T}(\alpha)$ & $\begin{array}{l}\text { Temporal correlation matrix (temporal correlation } \\
\text { coefficient) }\end{array}$ \\
\hline $\mathbf{P}_{\mathbf{s}}, \sigma_{\mathbf{w}}^{2}$ & $\begin{array}{l}\text { Respectively, the signal power factor and the noise } \\
\text { variance }\end{array}$ \\
\hline $\mathbf{G}_{\mathbf{s}}(\beta)$ & Pulse shaping matrix (roll-off factor) \\
\hline$\Lambda_{\tau}\left(\lambda_{n}\right)$ & $\begin{array}{l}\text { Diagonal matrix that models delay dispersion (coher- } \\
\text { ence bandwidth) }\end{array}$ \\
\hline $\mathbf{b}_{\phi}(\rho)$ & $\begin{array}{l}\text { LOS expected spatial signature (spatial correlation } \\
\text { vector) }\end{array}$ \\
\hline$\alpha_{\mathbf{t}}(\alpha)$ & $\begin{array}{l}\text { LOS expected temporal vector (temporal correlation } \\
\text { coefficient) }\end{array}$ \\
\hline $\mathbf{g}^{\left(\mathbf{k}_{\mathbf{0}}\right)}$ & Pulse shape vector for the first arrival \\
\hline
\end{tabular}

Description of notations frequently used within the text consecutive channel estimates:

$$
y^{(j)}(t)=\sum_{i=1}^{N_{\mathrm{paths}}} b_{i j}(t) \gamma_{i}(t) x\left(t-\tau_{i}\right) e^{j 2 \pi f_{i} t}+n^{(j)}(t)
$$

The $i$-th index discriminates the component within the multipath, and $N_{\text {paths }}$ is the number of impinging paths at the receiver. The transmitted signal $x(t)$ corresponds to the convolution of a pseudo-noise sequence $p(n)$ with the symmetric pulse shape $g(t)$ :

$$
x(t)=\sum_{n} g(t-n T) p(n)
$$

where $T$ is the symbol time. A correlator estimates the channel from the received signal $y^{(j)}(t)$ at each sensor $j$, and temporal lag $s$, with the help of the pseudo-noise sequence $p(n)$ of $N_{p}$ symbols,

$$
z_{s}^{(j)}(t)=\frac{1}{N_{p}} \sum_{n} y^{(j)}\left(t+\tau_{s}+n T\right) p^{*}(n)
$$

where $z_{s}^{(j)}(t)$ corresponds to the estimated channel coefficient at $j$-th sensor and $s$-th lag, as a function of time. Replacing (3) in (5), assuming zero mean noise, using the fact that the sequence $p(n)$ has unit power and is temporally uncorrelated, and the assumption that within $N_{p}$ symbols the steering coefficient and the path attenuation factor remain constant, and by discarding some cumbersome algebraic details, (5) becomes (6):

$$
z_{s}^{(j)}(t)=\sum_{i=1}^{N_{\text {paths }}} b_{i j}(t) \gamma_{i}(t) e^{j 2 \pi f_{i} t} g\left(t-\tau_{i}+\tau_{s}\right)+w_{s}^{(j)}(t)
$$

The Eq. (6) above shows that the estimated channel is obtained synchronously with the transmission time; therefore, we can assume $t=k T_{s}$ and hence temporal variation of $z_{s}^{(j)}(t)$ depends on $k$ in (7) and also Doppler frequencies are re-scaled:

$$
z_{s}^{(j)}(k)=\sum_{i=1}^{N_{\text {paths }}} b_{i j}(k) \gamma_{i}(k) e^{j 2 \pi f_{i} T_{s} k} g\left(\tau_{s}-\tau_{i}\right)+w_{s}^{(j)}(k)
$$

The residual noise component $w_{s}^{(j)}(t)$ is given by (8) and may be modeled as a zero mean complex white Gaussian random process (AS4).

$$
w_{s}^{(j)}(k)=\frac{1}{N_{p}} \sum_{k} n^{(j)}\left(k T_{s}+\tau_{s}+k T\right) p^{*}(k)
$$

Furthermore, taking into consideration that the multipath signal in our model has an exponential distribution and that the observation window is large enough to capture most of the energy from this scattered signal, and also considering just one arrival per lag, $N_{\text {paths }}$ in 
(7) has been set as equal to the number of lags $N$ in the observation window. Therefore, by stacking the channel coefficients at $s$-th lag described by (7), the channel vector estimation at sensor $j$ and at slot $k$, results in $\mathbf{z}^{(j)}(k)$. This vector may be expressed in terms of the shaping pulse and the noise estimation vector $\mathbf{w}^{(j)}(k)$ as in (9),

$$
\mathbf{z}^{(j)}(k)=\mathbf{G}_{s} \mathbf{b}^{(j)}(k)+\mathbf{w}^{(j)}(k)
$$

where the $i$-th element of vector $\mathbf{b}^{(j)}(k)$ contains $b^{(i j)}(k)$. $\gamma_{i}(k) \cdot \exp \left(j 2 \pi f_{i} T_{s} k\right)$ and the $i$-th column of the $N \times N$ square matrix $\mathbf{G}_{s}$ contains the shaping pulse delayed by $\tau_{i}$ samples:

$$
\mathbf{G}_{s}(\beta)=\frac{1}{\sqrt{T_{S}(1-\beta / 4)}}\left[\begin{array}{llll}
\mathbf{g}_{\mathbf{s}_{1}} & \mathbf{g}_{\mathbf{s}_{2}} & \ldots & \mathbf{g}_{\mathbf{s}_{\mathbf{N}}}
\end{array}\right]
$$

Observe that each one of the pulse shape vectors, $\mathbf{g}_{s i}$, in (10) may be modeled as in (11), where its elements $g_{k=s-i}$ refer to the shaping pulse sampled at $g\left(\tau_{s}-\tau_{i}\right)$ as described by (12):

$$
\begin{gathered}
\mathbf{g}_{\mathbf{s}_{i}}^{T}=\left[\begin{array}{ccccc}
g_{1-i} & \cdots & 1 & \cdots & g_{N-i}
\end{array}\right] \\
i \text { th element } \\
g_{k}=\frac{\operatorname{sinc}\left(\frac{k}{N_{s p c}}\right) \cos \left(\frac{\pi \beta k}{N_{s p c}}\right)}{1-\left(2 \beta k / N_{s p c}\right)^{2}}
\end{gathered}
$$

$N_{s p c}$ corresponds to the number of acquired samples per chip time. The length $N$ of vectors in (9) and (11) is the number of lags in channel estimates.

From (9), we can compute correlation matrix for two channel estimates obtained from slots $k$ and $m$, and sensors $j, j^{\prime}$, as in (13):

$$
\begin{aligned}
E\left\{\mathbf{z}^{(j)}(k) \mathbf{z}^{\left(j^{\prime}\right) H}(m)\right\} & =\mathbf{G}_{s} E\left\{\mathbf{b}^{(j)}(k) \mathbf{b}^{\left(j^{\prime}\right)^{H}}(m)\right\} \mathbf{G}_{s}^{T}+\sigma_{w}^{2} \mathbf{I}_{N} \\
& =\rho_{i j^{\prime}} \alpha^{k-m} \mathbf{G}_{s}(\beta) \mathbf{\Lambda}_{\tau} \mathbf{G}_{s}^{T}(\beta)+\sigma_{w}^{2} \mathbf{I}_{N}
\end{aligned}
$$

where $\beta$ is the roll-off factor shaping the transmission pulse, and $\boldsymbol{\Lambda}_{\tau}$ is a diagonal matrix that models signal temporal dispersion and its exponential power contribution. The last factorization is possible under the assumption of statistical independence for angular and temporal dispersion processes (AS 1) and also for multipath propagation and Doppler shift mechanisms. In fact, the $i, l$ element of the signal correlation matrix

$$
\begin{aligned}
E\left\{\mathbf{b}^{(j)}(k) \mathbf{b}^{\left.\left(j^{\prime}\right)^{H}(m)\right\}_{i, l}=}\right. & \left\{E \left\{b_{i j}(k) \gamma_{i}(k) e^{j 2 \pi f_{i} T_{s} k} .\right.\right. \\
& \left.\cdot b_{l j^{\prime}}^{*}(m) \gamma_{l}(m) e^{-j 2 \pi f_{l} T_{s} m}\right\} \\
E\left\{\mathbf{b}^{(j)}(k) \mathbf{b}^{\left.\left(j^{\prime}\right)^{H}(m)\right\}_{i, l}=}\right. & \rho_{i j^{\prime}} r_{i l} \alpha^{k-m} \delta_{i l}
\end{aligned}
$$

adopts the definitions:

$$
\begin{aligned}
E\left\{b_{i j}(k) b_{l j^{\prime}}^{*}(m)\right\} & =\rho_{j j^{\prime}} ; \\
E\left\{e^{j 2 \pi T_{s}\left(f_{i} k-f_{l} m\right)}\right\} & =\alpha^{k-m} ; \\
E\left\{\gamma_{i}(k) \gamma_{l}(m)\right\} & =r_{i l} \delta_{i l} \\
r_{i i}=E\left\{\gamma_{i}(k) \gamma_{i}(m)\right\} & =P_{s} e^{-\lambda_{n}\left(i-k_{0}\right)} u\left(i-k_{0}\right)
\end{aligned}
$$

where $\rho_{i j j^{\prime}}$ refers to the correlation between signatures at sensors $j$ and $j^{\prime}$; $\alpha$ refers to temporal correlation between channel estimates in two consecutive slots when temporal variation has been modeled as a first-order AR Markov process (AS 7); and $r_{i l}$ refers to the correlation between delays in lags $i$ and $l$, and $k_{0}$ refers to the TOA of the first path.

In particular, $r_{i l}$ is zero for paths at different lags since they fade independently and are assumed to be uncorrelated. Furthermore, the form of $r_{i i}$ in (15) responds to the assumption of having an exponential power delay profile (AS 6) with parameter $\lambda_{n}$, and it is very suitable for a NLOS condition.

Additionally, if vectors are arranged as

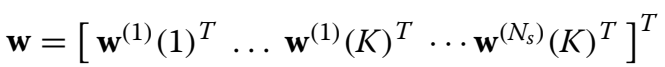

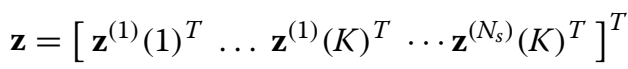

both signal and noise components may be described as temporally stationary, complex Gaussian random processes with certain means and correlation matrices. Noise is zero mean, temporally uncorrelated and independent of the propagation channel vectors and of variance $\sigma_{w}^{2}$.

When estimates in $\mathbf{z}$ are achieved under an NLOS condition, channel angular spread will tend to increase $[15,38]$. Such is the case, for instance, of a receiver at a mobile station (MS) in an urban environment. In this case, propagation is Rayleigh [35], and $\mathbf{z}$ may also be modeled as zero mean with correlation matrix $\mathbf{R}_{z}$. The general case for the model, however, corresponds to have LOS and Rice propagation [58]. In this case, the mean vector, $\mu_{\mathbf{z}}$, is not null. It could be the case for a receiver at the base station (BS) in a suburban environment. Therefore, we can model noise and signal as in (17):

$$
\mathbf{w} \sim C N\left(\mathbf{0}, \sigma_{w}^{2} \mathbf{I}\right), \quad \mathbf{z} \sim C N\left(\mu_{\mathbf{z}}, \mathbf{R}_{z}\right)
$$

The correlation matrix for channel estimates,

$$
\mathbf{R}_{\mathbf{z}}=E\left\{\mathbf{z z}^{H}\right\}
$$

is related to channel estimates at different slots, sensors and lags.

The correlation matrix, $\mathbf{R}_{z}$, may be written in the form

$$
\mathbf{R}_{\mathbf{z}}=\mathbf{R}_{\phi}(\rho) \otimes \mathbf{T}(\alpha) \otimes P_{s} \mathbf{G}_{s}(\beta) \boldsymbol{\Lambda}_{\tau}\left(\lambda_{n}\right) \mathbf{G}_{s}{ }^{H}(\beta)+\sigma_{w}^{2} \mathbf{I}
$$

in terms of their temporal and spatial components $[36,58]$. In this expression, the dispersed signal power fac- 
tor, $P_{s}$, refers to the variance of the received estimated path-power for first arrival from temporally dispersed signal in the case of Rayleigh propagation. Additionally, the temporal correlation matrix, $\mathbf{T}(\alpha)$, takes into consideration the temporal variation for the channel, and it is assumed to be equal for all delays; the spatial correlation matrix, $\mathbf{R}_{\phi}(\rho)$, contains the correlation coefficients for signatures between sensors; and $\otimes$ denotes the Kronecker product [59].

The exponential model used for delays is usually proposed in channel models, and it is given by

$$
\left\{\boldsymbol{\Lambda}_{\tau}\right\}_{i, i}=\exp \left[-\left(i-k_{0}\right) \lambda_{n}\right] u\left(i-k_{0}\right)
$$

in terms of both, the first arrival position $k_{0}$, and the dimensionless parameter $\lambda_{n}$. This latter is inversely related to delay spread normalized by the symbol time, and therefore it is closely related to channel coherence bandwidth $[47,48]$. In the following, $\lambda_{n}$ will be called the normalized coherence bandwidth.

The spatial correlation matrix, $\mathbf{R}_{\phi}(\rho)$ is modeled as

$$
\mathbf{R}_{\phi}(\rho)=\left[\begin{array}{cccc}
1 & \rho_{12} & \cdots & \rho_{1 N_{s}} \\
\rho_{12}^{*} & 1 & \cdots & \rho_{2 N_{s}} \\
\vdots & \vdots & \ddots & \vdots \\
\rho_{1 N_{s}}^{*} & \rho_{2 N_{s}}^{*} & \cdots & 1
\end{array}\right]
$$

where dependency with the source mean bearing and its angular spread meets through the correlation vector $\rho$, as will be explained later in (27)

$\mathbf{T}(\alpha)$ is modeled as a first-order AR Markov process,

$$
\mathbf{T}(\alpha)=\left[\begin{array}{ccccc}
1 & \alpha & \alpha^{2} & \cdots & \alpha^{K-1} \\
\alpha & 1 & & \ddots & \alpha^{K-2} \\
\alpha^{2} & & \ddots & & \\
\vdots & \ddots & & \\
\alpha^{K-1} & & & & 1
\end{array}\right]
$$

$\alpha$ is the temporal correlation coefficient between two consecutive vector samples, and $\rho_{i j}$ is the spatial correlation coefficient between sensors $i$ and $j$. Note also that $\mathbf{G}_{\mathbf{s}}$ is proportional to the identity matrix when sampling at the symbol rate.

Regarding the temporal correlation between consecutive estimates, the channel vector correlation matrix may be modeled as a Fully Coherent Dispersed (FCD) Source, a Partially Coherent Dispersed (PCD) Source, or an Incoherent Dispersed (ICD) Source. The general case corresponds to PCD, FCD being a particular case where estimates are completely correlated $(\alpha=1)$, and ICD the case where estimates are uncorrelated $(\alpha=0)[35,36]$.

In the case of Rice propagation, the first arrival has a non-null mean and disturbs the exponential distribution for delays. Expression (9) turns into (23), where $f_{0}$ is the Doppler frequency for the LOS component, and $\mathbf{g}^{\left(k_{0}\right)}$ identifies the pulse shape vector for this arrival:

$$
\begin{aligned}
\mathbf{z}^{(j)}(k)= & b_{0 j}(k) \gamma_{0}(k) e^{j 2 \pi f_{0} T_{s} k} \mathbf{g}^{\left(k_{0}\right)} \\
& +\mathbf{G}_{s} \mathbf{b}^{(j)}(k)+\mathbf{w}^{(j)}(k)
\end{aligned}
$$

Note that the right part of the summation in (23) corresponds to the dispersed NLOS signal and has a null expected value. Moreover, since delay dispersion and Doppler are assumed independent, the mean channel gain is computed as follows in (24) where the time dependency of the steering vector has been discarded since its value is expected to remain unchanged for the LOS path along the position acquisition (AS 5).

$$
\begin{aligned}
E\left\{\mathbf{z}^{(j)}(k)\right\} & =E\left\{b_{0 j} \gamma_{0}(k) e^{j 2 \pi f_{0} T_{s} k} \mathbf{g}^{\left(k_{0}\right)}\right\} \\
& =A_{0} E\left\{b_{0 j}\right\} E\left\{e^{j 2 \pi f_{0} T_{s} k}\right\} \mathbf{g}^{\left(k_{0}\right)}
\end{aligned}
$$

where $A_{0}$ corresponds to the mean signal level for the LOS component. If $\mu_{\mathbf{z}}$ denotes the mean channel vector arranged as $\mathbf{z}$ and $\mathbf{w}$, in (16), it could be expressed in terms of the spatial signature for the LOS component $\mathbf{b}_{\phi}$, the expected Doppler vector $\alpha_{t}$, and the pulse shape vector for first arrival $\mathbf{g}^{(k 0)}$, as

$$
\mu_{\mathbf{z}}=E\{\mathbf{b}\} \otimes E\left\{e^{j 2 \pi f_{0} T_{s} k}\right\} \otimes A_{0} \mathbf{g}^{\left(k_{0}\right)}
$$

The spatial signature described by the LOS component when a uniform linear array (ULA) is used may be computed geometrically using a signal angular distribution [60], as in

$$
\begin{aligned}
\mathbf{b}_{\phi} & =E\{\mathbf{b}\} \\
\operatorname{with}[E\{\mathbf{b}\}]_{n} & =E\left\{b_{n}\right\} \\
& =\frac{1}{\sqrt{2 \pi} \Delta_{\phi}} \int_{-\pi}^{\pi} e^{-\frac{\left(\phi-\phi_{0}\right)^{2}}{2 \Delta_{\phi}^{2}}} e^{-j n \pi \sin (\phi)} d \phi
\end{aligned}
$$

In this case, spatial distribution is modeled as Gaussian (AS 5), centered around $\phi_{0}$, with angular spread $\Delta_{\phi}$ and subscript $n$ corresponds to the sensor position for $n \in\left[0, N_{s-1}\right]$. The angular spread corresponds to the standard deviation of the direction of arrivals from multipath components at the receiver when a normalized version of the PAS is used as the weighting function. Some works report that Laplacian distribution can provide a good match to this angular distribution $[15,53,61]$; but when it is used in (27) instead of a Gaussian distribution, meaningless variations are achieved.

Remembering that the correlation matrix $\mathbf{R}_{\phi}$ described in (27) is related to (26), the expected spatial signature in (28) results. 


$$
\begin{aligned}
{\left[\mathbf{R}_{\phi}\right]_{n_{1}, n_{2}} } & =E\left\{b_{n_{1}} b_{n_{2}}^{*}\right\} \\
\text { with } E\left\{b_{n_{1}} b_{n_{2}}^{*}\right\} & =\frac{1}{\sqrt{2 \pi} \Delta_{\phi}} \int_{-\pi}^{\pi} e^{-\frac{\left(\phi-\phi_{0}\right)^{2}}{2 \Delta_{\phi}^{2}}} e^{-j\left(n_{1}-n_{2}\right) \pi \sin (\phi)} d \phi
\end{aligned}
$$

$$
\mathbf{b}_{\phi}(\rho)=\left[\begin{array}{lllll}
1 & \rho_{12} & \rho_{13} & \ldots & \rho_{1 N_{s}}
\end{array}\right]^{H}
$$

Note from (25) and from the fact that temporal variation due to Doppler may again be modeled as a first-order AR Markov process (AS 7), as the temporal vector, $\alpha_{t}(\alpha)$, is a function of $\alpha$, and it takes the form

$$
\alpha_{t}(\alpha)=\left[\begin{array}{lllll}
1 & \alpha & \alpha^{2} & \ldots & \alpha^{K-1}
\end{array}\right]^{T}
$$

Therefore (25) becomes:

$$
\mu_{\mathbf{z}}=\mathbf{b}_{\phi}(\rho) \otimes \alpha_{t}(\alpha) \otimes A_{0} \mathbf{g}^{\left(k_{0}\right)}(\beta)
$$

\subsection{Computing the Cramer-Rao bounds for delay estimates}

Highlighting the importance of Cramer-Rao bound not only as a means to quantify errors from a set of parameters to be estimated but also as a modeling tool since it allows for the evaluation of the impact of various parameters in the estimation error, we will continue with the derivation of this bound for our model, introduced mainly in (17), (19), and (30). Consequently, the following parameter vector is defined in (31), where $k_{0}$ is the time of arrival normalized for the chip time, $\lambda_{n}$ is the normalized coherence bandwidth, $\rho$ is a vector containing the real and imaginary parts of the complex correlation coefficients among sensors, and the remaining parameters have previously been defined. All of them except $k_{0}$ are nuisance parameters.

$$
\begin{aligned}
\boldsymbol{\Psi} & =\left[k_{0}, \lambda_{n}, \beta, P_{s}, \sigma_{w}^{2}, \alpha, \rho^{T}, A_{0}\right]^{T} \\
\rho & =\left[\rho_{1, \operatorname{Re}}, \rho_{2, \operatorname{Re}}, \ldots, \rho_{N_{c}, \operatorname{Re}}, \rho_{1, \mathrm{Im}}, \rho_{2, \mathrm{Im}}, \ldots, \rho_{N_{c}, \mathrm{Im}}\right]^{T}
\end{aligned}
$$

Note that in case of a Rayleigh fading channel, there is not a dominant LOS path and therefore $A_{0}$ is zero and may be discarded, reducing the parameter vector to (32).

$$
\begin{aligned}
\boldsymbol{\Psi} & =\left[k_{0}, \lambda_{n}, \beta, P_{s}, \sigma_{w}^{2}, \alpha, \rho^{T}\right]^{T} \\
\rho & =\left[\rho_{1, \operatorname{Re}}, \rho_{2, \operatorname{Re}}, \ldots, \rho_{N_{c}, \operatorname{Re}}, \rho_{1, \operatorname{Im}}, \rho_{2, \operatorname{Im}}, \ldots, \rho_{N_{c}, \operatorname{Im}}\right]^{T}
\end{aligned}
$$

Since channel vector estimates being stacked in $\mathbf{z}$ are assumed complex Gaussian distributed, the probability density function for $\mathbf{z}$ is expressed as in (33), and the Cramer-Rao bounds for the parameters in (31) correspond to the diagonal elements within the inverse of the FIM. Furthermore, FIM elements for the Rice LOS model may be expressed as it is seen in (34) [26] and in the case of Rayleigh fading as given in Eq. (35). Also note that $\mathbf{R}_{z}$ corresponds to the covariance matrix for the general Rice case in (34) and it is equal to the correlation matrix for the Rayleigh case in (35) since the mean is null for this latter case.

$$
p(\mathbf{z})=\frac{1}{\pi^{K \cdot N_{s} \cdot N} \operatorname{det}\left(\mathbf{R}_{z}\right)} \exp \left[-\left(\mathbf{z}-\mu_{z}\right)^{H} \mathbf{R}_{z}^{-1}\left(\mathbf{z}-\mu_{z}\right)\right]
$$

$$
\begin{aligned}
{\left[\mathbf{F}_{\mathbf{\Psi}}^{L O S}\right]_{p q}=} & -E\left[\frac{\partial^{2} \ln \{p(\mathbf{z} ; \mathbf{\Psi})\}}{\partial \boldsymbol{\Psi}_{p} \partial \boldsymbol{\Psi}_{q}}\right] \\
= & \operatorname{tr}\left(\mathbf{R}_{\mathbf{z}}^{-1} \frac{\partial \mathbf{R}_{\mathbf{z}}}{\partial \mathbf{\Psi}_{p}} \mathbf{R}_{\mathbf{z}}^{-1} \frac{\partial \mathbf{R}_{\mathbf{z}}}{\partial \boldsymbol{\Psi}_{q}}\right) \\
& +2 \operatorname{Re}\left(\frac{\partial \mu_{\mathbf{z}}^{H}}{\partial \mathbf{\Psi}_{p}} \mathbf{R}_{\mathbf{z}}^{-1} \frac{\partial \mu_{\mathbf{z}}}{\partial \boldsymbol{\Psi}_{q}}\right) \\
{\left[\mathbf{F}_{\mathbf{\Psi}}\right]_{p q}=} & -E\left[\frac{\partial^{2} \ln \{p(\mathbf{z} ; \boldsymbol{\Psi})\}}{\partial \boldsymbol{\Psi}_{p} \partial \mathbf{\Psi}_{q}}\right] \\
= & \operatorname{tr}\left(\mathbf{R}_{\mathbf{z}}^{-1} \frac{\partial \mathbf{R}_{\mathbf{z}}}{\partial \mathbf{\Psi}_{p}} \mathbf{R}_{\mathbf{z}}^{-1} \frac{\partial \mathbf{R}_{\mathbf{z}}}{\partial \mathbf{\Psi}_{q}}\right)
\end{aligned}
$$

\subsubsection{Cramer-Rao bounds for the NLOS Rayleigh fading model}

It will be shown below that (35) becomes (36) when $\mathbf{R}_{z}^{-1}$ and their partial derivatives are computed and replaced in the expression above.

$$
\begin{aligned}
\mathbf{F}_{\Psi}= & \sum_{k=1}^{N s} \sum_{k_{1}=1}^{K} \mathbf{G}_{k, k_{1}} \mathbf{J}_{\Psi^{\prime}} \mathbf{G}_{k, k_{1}}^{T}+C_{1} \mathbf{e}_{N p}^{(6)} \mathbf{e}_{N p}^{(6)}+ \\
& +\sum_{q_{1}=1}^{2 N} \sum_{q 2=1}^{2 N} C_{2}^{\left(q_{1}, q_{2}\right)} \mathbf{e}_{N p}^{\left(6+q_{1}\right)} \mathbf{e}_{N p}^{\left(6+q_{2}\right)^{T}}
\end{aligned}
$$

Expression (36) illustrates the way that the required FIM $\left(F_{\Psi}\right)$ gains information from the contribution of each available channel estimate through the eigenvalues from both the temporal- and spatial- correlation matrices. In fact, $\mathbf{G}_{k, k 1}$ matrix, and $C_{1}$ and $C_{2}^{(q 1, q 2)}$ coefficients allow this update in a computationally efficient manner. $\mathbf{G}_{k, k 1}$ has a global impact since it weighs the partial FIMs $\left(\mathbf{J}_{\Psi^{\prime}}\right)$ computed at each new iteration by taking advantage of the structure of the power delay profile in $\Lambda_{\tau}$. On the other side, $C_{1}$ refers to the diagonal term for the parameter $\alpha$, and $C_{2}^{\left(q_{1}, q_{2}\right)}$ to the crossed terms related with the correlation coefficients of the spatial correlation matrix $\mathbf{R}_{\phi}$. Terms in (36) are defined in expressions (37)-(43). In particular, it is a worth noting that a singular value decomposition has been performed over the temporal correlation matrix $\mathbf{T}$, and over the spatial correlation matrix $\mathbf{R}_{\phi}$, as it is shown in (37), being $\lambda_{t}^{(k)}$ and $\lambda_{\phi}^{\left(k_{1}\right)}$ the eigenvalues of $\mathbf{T}$ and $\mathbf{R}_{\phi}$ respectively. Similarly, $\mathbf{u}_{t}^{(k)}$ and $\mathbf{u}_{\phi}^{\left(k_{1}\right)}$ correspond to the eigenvectors of these correlation matrices. $N_{c}$ in (37) 
is the number of parameters associated with the spatial correlation matrix and therefore depends on the array size $N_{s}$, with $N_{p}$ being the total number of parameters in our model and $K$ the number of channel vector estimates.

$$
\begin{aligned}
\mathbf{T} & =\mathbf{U}_{t} \mathbf{\Lambda}_{t} \mathbf{U}_{t}^{\mathbf{H}}, \mathbf{U}_{t}=\left[\mathbf{u}_{t}^{(1)}, \mathbf{u}_{t}^{(2)}, \ldots, \mathbf{u}_{t}^{(K)}\right] \\
\mathbf{\Lambda}_{t} & =\operatorname{diag}\left[\lambda_{t}^{(1)}, \lambda_{t}^{(2)}, \ldots, \lambda_{t}^{(K)}\right] \\
\mathbf{R}_{\phi} & =\mathbf{U}_{\phi} \boldsymbol{\Lambda}_{\phi} \mathbf{U}_{\phi}^{\mathbf{H}}, \mathbf{U}_{\phi}=\left[\mathbf{u}_{\phi}^{(1)}, \mathbf{u}_{\phi}^{(2)}, \ldots, \mathbf{u}_{\phi}^{(N)}\right] \\
\mathbf{\Lambda}_{\phi} & =\operatorname{diag}\left[\lambda_{\phi}^{(1)}, \lambda_{\phi}^{(2)}, \ldots, \lambda_{\phi}^{(N s)}\right] \\
\mathbf{e}_{v}^{(q)} & =[0, \ldots, 0,1,0, \ldots, 0]_{v}^{T} \\
& \stackrel{\uparrow}{q} \text { th } \quad \text { element } \\
N_{p} & =6+2 N_{c} ; \quad N_{c}=N_{s}\left(N_{s}-1\right) / 2
\end{aligned}
$$

See in (38) as $\boldsymbol{\Psi}^{\prime}$ differs for each new $k$ and $k_{1}$ since the parameter $\gamma_{k, k 1}$ in (40) refers to the signal power weighted by the respective spatial and temporal eigenvalues. $\mathbf{G}_{k, k 1}$ also depends on the partial derivatives of these eigenvalues as related to the temporal correlation factor $\alpha$ and from the spatial correlation coefficients in $\rho$.

$\boldsymbol{\Psi}^{\prime}=\left[k_{0}, \lambda_{n}, \beta, \gamma_{k, k_{1}}, \sigma_{w}^{2}\right]^{T} \mathbf{G}_{k, k_{1}}=\left[\begin{array}{ccccc}1 & 0 & 0 & 0 & 0 \\ 0 & 1 & 0 & 0 & 0 \\ 0 & 0 & 1 & 0 & 0 \\ 0 & 0 & 0 & \lambda_{\phi}^{(k)} \lambda_{t}^{\left(k_{1}\right)} & 0 \\ 0 & 0 & 0 & 0 & 1 \\ 0 & 0 & 0 & P_{s} \lambda_{\phi}^{(k)} \frac{\partial \lambda_{t}^{\left(k_{1}\right)}}{\partial \alpha} & 0 \\ 0 & 0 & 0 & P_{s} \frac{\partial \lambda_{\phi}^{(k)}}{\partial \rho} \lambda_{t}^{\left(k_{1}\right)} & 0\end{array}\right]_{\left(6+2 N_{c}\right) x 5}$

These derivatives are described in (39) and (40).

$\frac{\partial \lambda_{\phi}^{(k)}}{\partial \rho}=\left[\frac{\partial \lambda_{\phi}^{(k)}}{\partial \rho_{1, \operatorname{Re}}}, \frac{\partial \lambda_{\phi}^{(k)}}{\partial \rho_{2, \operatorname{Re}}}, \ldots, \frac{\partial \lambda_{\phi}^{(k)}}{\partial \rho_{N_{c}, \operatorname{Re}}}, \frac{\partial \lambda_{\phi}^{(k)}}{\partial \rho_{1, \mathrm{Im}}}, \frac{\partial \lambda_{\phi}^{(k)}}{\partial \rho_{2, \mathrm{Im}}}, \ldots, \frac{\partial \lambda_{\phi}^{(k)}}{\partial \rho_{N_{c}, \mathrm{Im}}}\right]^{T}$

$$
\begin{aligned}
\gamma_{k, k_{1}} & =\lambda_{\phi}^{(k)} \lambda_{t}^{\left(k_{1}\right)} P_{s} & \\
\dot{\lambda}_{t}^{\left(k_{1}\right)} & =\frac{d \lambda_{t}^{\left(k_{1}\right)}}{d \alpha} & \dot{\mathbf{u}}_{t}^{\left(k_{1}\right)}=\frac{d \mathbf{u}_{t}^{\left(k_{1}\right)}}{d \alpha}
\end{aligned}
$$

The partial FIMs required in (36) are described as in (41), where the partial correlation matrix $\mathbf{R}_{k, k 1}$ takes the form in (42).

$$
\begin{aligned}
\left\{\mathbf{J}_{\Psi^{\prime}}\right\}_{p q} & =\operatorname{tr}\left(\mathbf{R}_{k, k_{1}}^{-1} \frac{\partial \mathbf{R}_{k, k_{1}}}{\partial \mathbf{\Psi}_{p}^{\prime}} \mathbf{R}_{k, k_{1}}^{-1} \frac{\partial \mathbf{R}_{k, k_{1}}}{\partial \mathbf{\Psi}_{q}^{\prime}}\right) \\
\mathbf{R}_{k, k_{1}} & =P_{s} \lambda_{\phi}^{(k)} \lambda_{t}^{\left(k_{1}\right)} \mathbf{G}_{s} \boldsymbol{\Lambda}_{\tau} \mathbf{G}_{s}^{T}+\sigma_{w}^{2} \mathbf{I}_{N}
\end{aligned}
$$

$C_{1}$ and $C_{2}^{\left(q_{1}, q_{2}\right)}$ coefficients are described in (43).

$$
\begin{aligned}
C_{1}= & -P_{s}^{2} \sum_{k=1}^{N s} \sum_{k_{1}=1}^{K} \sum_{l_{1}=1}^{K}\left[\lambda_{\phi}^{(k)^{2}}\left(\lambda_{t}^{\left(k_{1}\right)}-\lambda_{t}^{\left(l_{1}\right)}\right)^{2} \mathbf{u}_{t}^{\left(k_{1}\right)^{H}} \dot{\mathbf{u}}_{t}^{\left(l_{1}\right)} \mathbf{u}_{t}^{\left(l_{1}\right)^{H}} \dot{\mathbf{u}}_{t}^{\left(k_{1}\right)} .\right. \\
C_{2}^{\left(q_{1}, q_{2}\right)}= & \left.\left.-P_{s}^{2} \sum_{k=1}^{N s} \sum_{l=1}^{N s} \sum_{k, k_{1}}^{N} \mathbf{G}_{s} \boldsymbol{\Lambda}_{\tau} \mathbf{G}_{s}^{T} \mathbf{R}_{k, l_{1}}^{-1} \mathbf{G}_{s} \mathbf{\Lambda}_{\tau} \mathbf{G}_{s}^{T}\right\}\right] \\
& \times\left(\lambda_{t}^{\left(k_{1}\right)^{2}}\left(\lambda_{\phi}^{(k)}-\lambda_{\phi}^{(l)}\right)^{2}\left(\mathbf{u}_{\phi}^{(l)} \frac{\partial \mathbf{u}_{\phi}^{(k)}}{\partial \rho_{q_{2}}}\right) \cdot \operatorname{tr}\left\{\mathbf{u}_{\phi}^{(l)} \partial \mathbf{R}_{k, k_{1}}^{-1} \mathbf{G}_{s} \mathbf{\Lambda}_{\tau} \mathbf{G}_{s}^{T} \mathbf{R}_{l, k_{1}}^{-1} \mathbf{G}_{s} \mathbf{\Lambda}_{\tau} \mathbf{G}_{s}^{T}\right\}\right]
\end{aligned}
$$

Derivations are certainly quite algebraically extensive, and their main steps will be commented on briefly.

First of all, $\mathbf{R}_{z}^{-1}$ is expressed as in (44) by using the Kronecker product properties [59], with $\mathbf{R}_{k, k 1}$ defined as in (42):

$\mathbf{R}_{\mathbf{z}}^{-1}=\sum_{k=1}^{N_{s}} \mathbf{u}_{\phi}^{(k)} \mathbf{u}_{\phi}^{(k) H} \otimes \sum_{k_{1}=1}^{K} \mathbf{u}_{k}^{\left(k_{1}\right)} \mathbf{u}_{k}^{\left(k_{1}\right) H} \otimes \sum_{k_{2}=1}^{N} \mathbf{e}_{N}^{\left(k_{2}\right)} \mathbf{e}_{N}^{\left(k_{2}\right) H} \mathbf{R}_{k, k_{1}}^{-1}$

Derivatives required in (35) must also be computed, and it is easy to show how they take the form described in Eq. (45), being $\mathbf{A}, \mathbf{B}$, and $\mathbf{C}$ the matrices exhibited in Table 2.

$$
\frac{\partial \mathbf{R}_{\mathbf{z}}}{\partial \mathbf{\Psi}_{p}}=\mathbf{A} \otimes \mathbf{B} \otimes \mathbf{C}
$$

For example, when a derivative relative to $k_{0}$ is required, the corresponding value of $p$ within the table is " 1 ," and therefore, the corresponding result is assembled as in (46).

$$
\partial \mathbf{R}_{\mathbf{z}} / \partial \boldsymbol{\Psi}_{p=1}=\partial \mathbf{R}_{\mathbf{z}} / \partial k_{0}=\mathbf{R}_{\phi} \otimes \mathbf{T}_{k} \otimes P_{s} \partial \boldsymbol{\Lambda}_{\tau} / \partial k_{0}
$$

From inserting Eqs. (44) and (45) in (35), and after some simplifications, (47) originates. Finally, by replacing

Table 2 Elements required in (45) to assemble the FIM in (35) for a Rayleigh fading channel

\begin{tabular}{lllll}
\hline$p$ & $\Psi_{p}$ & $\mathrm{~A}$ & $\mathrm{~B}$ & $\mathrm{C}$ \\
\hline 1 & $k_{0}$ & $\mathbf{R}_{\phi}$ & $\mathbf{T}_{\mathbf{K}}$ & $\mathbf{P}_{s} \partial \mathbf{\Lambda}_{\tau} / \partial k_{0}$ \\
2 & $\lambda_{n}$ & $\mathbf{R}_{\phi}$ & $\mathbf{T}_{\mathbf{K}}$ & $\mathbf{P}_{\mathbf{s}} \partial \mathbf{\Lambda}_{\tau} / \partial \mathbf{k}_{\mathbf{0}}$ \\
3 & $\beta$ & $\mathbf{R}_{\phi}$ & $\mathbf{T}_{\mathbf{K}}$ & $\mathbf{P}_{\mathbf{s}} \partial\left(\mathbf{G}_{\mathbf{s}} \mathbf{\Lambda}_{\tau} \mathbf{G}_{\mathbf{s}}^{\mathbf{T}}\right) / \partial \beta$ \\
4 & $P_{s}$ & $\mathbf{R}_{\phi}$ & $\mathbf{T}_{\mathbf{K}}$ & $\mathbf{G}_{\mathbf{s}} \mathbf{\Lambda}_{\tau} \mathbf{G}_{\mathbf{s}}^{\mathbf{T}}$ \\
5 & $\sigma_{w}^{2}$ & $\mathbf{I}_{\mathbf{N s}}$ & $\mathbf{I}_{\mathbf{K}}$ & $\mathbf{I}_{\mathbf{N}}$ \\
6 & $\alpha$ & $\mathbf{R}_{\phi}$ & $\partial \mathbf{T} / \partial \alpha$ & $\mathbf{P}_{\mathbf{s}} \mathbf{G}_{\mathbf{s}} \mathbf{\Lambda}_{\tau} \mathbf{G}_{\mathbf{s}}^{\mathbf{T}}$ \\
$7: N_{p}$ & $\rho_{p-6}$ & $\partial \mathbf{R}_{\phi} / \partial \rho_{\mathbf{p}-\mathbf{6}}$ & $\mathbf{T}_{\mathbf{K}}$ & $\mathbf{P}_{\mathbf{s}} \mathbf{G}_{\mathbf{s}} \mathbf{\Lambda}_{\tau} \mathbf{G}_{\mathbf{s}}^{\mathbf{T}}$ \\
\hline
\end{tabular}

General modeling of a NLOS dispersed signal 
the values from Table 2, as in the example above, and rearranging terms, expression (36) is reached.

$$
\begin{aligned}
{\left[\mathbf{F}_{\Psi}\right]_{p q}=} & \\
= & \sum_{k=1}^{N s} \sum_{l=1}^{N s} \mathbf{u}_{\phi}^{(k){ }^{H}} \mathbf{A}_{p} \mathbf{u}_{\phi}^{(l)} \mathbf{u}_{\phi}^{(l)} \mathbf{A}_{q} \mathbf{u}_{\phi}^{(k)} . \\
& \cdot \sum_{k_{1}=1}^{K} \sum_{l_{1}=1}^{K} \mathbf{u}_{K}^{\left(k_{1}\right)^{H}} \mathbf{B}_{p} \mathbf{u}_{K}^{\left(l_{1}\right)} \mathbf{u}_{K}^{\left(l_{1}\right)}{ }^{H} \mathbf{B}_{q} \mathbf{u}_{K}^{\left(k_{1}\right)} t r \\
& \times\left(\mathbf{R}_{k, k_{1}}^{-1} \mathbf{C}_{p} \mathbf{R}_{l, l_{1}}^{-1} \mathbf{C}_{q}\right)
\end{aligned}
$$

Furthermore, expression in (35) allows further simplifications when the sampling is performed at the chip rate. If it is the case, $\mathbf{G}_{s}$ in (19) becomes the identity matrix $\mathbf{I}$, and the roll-off factor may be discarded, reducing the number of parameters required to compute the Fisher matrix [36]. More details about these simplifications may be found in Section AF1.1 within Additional file 1.

\subsubsection{Cramer-Rao bounds for the LOS Rice fading model}

In case of an LOS condition, fading is Rice and therefore the mean channel vector estimate $\mu_{z} \mathrm{z}$ is not null, and it is described in (30) in terms of the expected spatial signature $\mathbf{b}_{\phi}$, the Doppler vector $\alpha_{t}$, the pulse shaping vector for the first arrival $\mathbf{g}^{\left(k_{0}\right)}$, and the mean signal level for the LOS component $A_{0}$. All these components were described in (21)-(29).

Since the mean channel vector is different to zero for the LOS case, the parameter vector is described in (31) and the computation of the FIM in (34) adds some derivatives that must also be computed. It is easy to show these take the form

$$
\frac{\partial \mu_{\mathbf{z}}}{\partial \boldsymbol{\Psi}_{p}}=\mathbf{D} \otimes \mathbf{E} \otimes \mathbf{F}
$$

being $\mathbf{D}, \mathbf{E}$, and $\mathbf{F}$, the vectors contained in Table 3. For instance, when a derivative relative to $k_{0}$ is required, the corresponding value of $p$ within the table is one, and the derivative in (48) becomes as in (49):

Table 3 Definition of elements in (48) required for additional derivatives in (34) when computing the FIM

\begin{tabular}{lllll}
\hline$p$ & $\Psi_{p}$ & $D$ & $E$ & $F$ \\
\hline 1 & $k_{0}$ & $\mathbf{b}_{\phi}$ & $\alpha_{\mathbf{t}}$ & $A_{0} \partial \mathbf{g}^{\left(k_{0}\right)} / \partial k_{0}$ \\
2 & $\alpha_{n}$ & - & - & - \\
3 & $\beta$ & $\mathbf{b}_{\phi}$ & $\alpha_{\mathbf{t}}$ & $A_{0} \partial \mathbf{g}^{\left(k_{0}\right)} / \partial \beta$ \\
4 & $P_{s}$ & - & - & - \\
5 & $\sigma_{w}^{2}$ & - & - & - \\
6 & $\alpha$ & $\mathbf{b}_{\phi}$ & $\partial \alpha_{\mathbf{t}} / \partial \alpha$ & $A_{0} \mathbf{g}^{k_{0}}$ \\
$7: N_{p-1}$ & $\rho_{p-6}$ & $\partial \mathbf{b}_{\phi} / \partial \rho_{\mathbf{p}-6}$ & $\alpha_{\mathbf{t}}$ & $A_{0} \mathbf{g}^{k_{0}}$ \\
$N_{p}$ & $A_{0}$ & $\mathbf{b}_{\phi}$ & $\alpha_{\mathbf{t}}$ & $\mathbf{g}^{k_{0}}$ \\
\hline
\end{tabular}

Modeling of a LOS Rice fading channel

$$
\partial \mu_{\mathbf{z}} / \partial \boldsymbol{\Psi}_{p=1}=\partial \mu_{\mathbf{z}} / \partial k_{0}=\mathbf{b}_{\phi} \otimes \alpha_{t} \otimes A_{o} \partial \mathbf{g}^{\left(k_{0}\right)} / \partial k_{0}
$$

It may be shown that (34) becomes (50) when $\mathbf{R}_{z}^{-1}$ in (49) and the partial derivatives in (48) are replaced within (34).

$\mathbf{F}_{\Psi}^{L O S}=\mathbf{F}_{\Psi}+2 \operatorname{Re}\left\{\begin{array}{l}\mathbf{1}_{N_{s}}^{T} \sum_{k=1}^{N_{s}} \mathbf{u}_{\phi}^{(\mathbf{k})} \mathbf{u}_{\phi}^{(\mathbf{k})^{H}} \odot \\ \odot \frac{\partial \mathbf{b}_{\mathbf{z}}}{\partial \mathbf{\Psi}_{p}} \frac{\partial \mathbf{b}_{\mathbf{z}}^{H}}{\partial \mathbf{\Psi}_{q}} \mathbf{1}_{N_{s}} \cdot \mathbf{1}_{K}^{T} \sum_{k_{1}=1}^{K} \mathbf{u}_{\mathbf{T}}^{\left(\mathbf{k}_{1}\right)} \mathbf{u}_{\mathbf{T}}^{\left(\mathbf{k}_{1}\right)^{H}} \odot \\ \odot \frac{\partial \alpha_{t}}{\partial \mathbf{\Psi}_{p}} \frac{\partial \alpha_{t}^{T}}{\partial \mathbf{\Psi}_{q}} \mathbf{1}_{K} \cdot \frac{\partial \mathbf{g}^{\left(k_{0}\right)^{T}}}{\partial \mathbf{\Psi}_{p}} \mathbf{R}_{k, k_{1}}^{-1} \frac{\partial \mathbf{g}^{\left(k_{0}\right)}}{\partial \mathbf{\Psi}_{q}}\end{array}\right\}$

In the expression above, $\mathbf{F}_{\Psi}$ corresponds to the FIM for the NLOS model in (36), but since the parameter $A_{0}$ was added for the LOS model, $\mathbf{G}_{k, k 1}$ in (38) must be replaced for $\mathbf{G}_{k, k 1}^{L O S}$ in (51). Furthermore, $\odot$ notes the Hadamard Product.

$$
\mathbf{G}_{k, k_{1}}^{L O S}=\left[\begin{array}{l}
\mathbf{G}_{k, k_{1}} \\
\mathbf{0}
\end{array}\right]_{\left(7+2 N_{c}\right) x 5}
$$

Computing the CRBs from the previous equations may be computationally expensive, especially when the number of available channel vector estimates $K$ is high. Furthermore, expressions for FCD sources require another more suitable factorization. Therefore, asymptotic expressions when $K$ is high and adequate expressions for FCD sources have been computed in $[35,36]$. See Sections AF1.2 and AF1.3 within Additional file 1.

\subsection{CRBs in timing estimation and the extent of positioning errors}

In order to put $\mathrm{CRB}$ results in relation to potential errors introduced in terms of distance range, (52) will be used, where $e$ corresponds to the range error estimation, $c$ to light speed, and $T_{c}$ to the system chip time.

$$
e=c \cdot \sqrt{\operatorname{CRB}\left(k_{0}\right)} T_{c}
$$

Consequently, an estimation error standard deviation of one chip time results in a range error in the order of $240 \mathrm{~m}$ for IS-95 and around $80 \mathrm{~m}$ for WCDMA since chip period is a little more than three times higher for IS-95 in relation to WCDMA. In the sequel, a WCDMA system will be referred to by default.

\subsection{Coherence bandwidth and CRBs}

As was mentioned previously, channel coherence bandwidth, $B_{c}$, is proportional to the normalized coherence bandwidth $\lambda_{n}$, and to the chip rate, $R_{c}$. The exact proportionality factor depends on the application but is lower than $1 /(2 \pi)[47,48]$; therefore, it will be set to $1 / 10$ as shown in (53), and the estimation error of this bandwidth may be related to the CRB for $\lambda_{n}$ as: 


$$
\begin{aligned}
& B_{c} \approx \frac{1}{10} \lambda_{n} R_{c} \\
& e_{B_{c}} \approx \frac{1}{10} R_{c} \sqrt{\operatorname{CRB}\left(\lambda_{n}\right)}
\end{aligned}
$$

Through the use of (54), it is easy to understand that error estimation for the coherence bandwidth is close to $1 \%$ of chip rate when the square root of the CRB is close to $1 / 10$. It would correspond for example to an uncertainty of around $38 \mathrm{kHz}$ for a WCDMA system and around of $12 \mathrm{kHz}$ for IS-95.

\subsection{Timing estimation: the minimum variance method}

This section introduces the minimum variance (MV) TOA estimator, a practical method available in the literature [49], in order to compare its behavior with that described by our CRBs.

Remembering that our data is a collection of $K$ channel vector estimates infected with noise, recorded in a time interval of duration $K T_{s}$ as follows:

$$
\begin{aligned}
y(\tau ; k)= & \sum_{i=1}^{L} a_{i}(k) g\left(\tau-\tau_{i}\right) \\
& +v(\tau ; k) ; \quad a_{i}(k), v(\tau ; k) \in C \quad \forall k=1, \ldots, K
\end{aligned}
$$

where $\tau_{i}$ and $a_{i}(t)$ refer respectively to the delays and the time-varying amplitudes of the $L$ propagation paths, $g(\tau)$ to the pulse shape, and $v(\tau ; n)$ to the noise which is assumed temporally, not correlated among successive slots $(n)$.

When the discrete Fourier transform (DFT) is computed from channel vector estimates, (56) results:

$$
y(w ; n)=\sum_{i=1}^{L} a_{i}(n) g(w) \exp \left(-j w \tau_{i}\right)+v(w ; n)
$$

And the delays' estimation problem turns into the estimation of the position of spectral lines. Stacking the samples of the transformed domain in a single vector, (56) may be rewritten as (57):

$$
\begin{aligned}
\mathbf{y}(n)= & {\left[\begin{array}{c}
y\left(\omega_{o} ; n\right) \\
y\left(\omega_{1} ; n\right) \\
\vdots \\
y\left(\omega_{M-1} ; n\right)
\end{array}\right]=\sum_{i=1}^{L} a_{i}(n) \mathbf{G e}_{\tau_{i}} } \\
& +\mathbf{v}(n)=\mathbf{G E}_{\tau} \mathbf{a}(n)+\mathbf{v}(n)
\end{aligned}
$$

where $\mathbf{G}$ is a diagonal matrix containing the DFT of the raised cosine pulse shaping filter and $\mathbf{E}_{\tau}$ is defined below:

$$
\begin{aligned}
\mathbf{E}_{\tau} & =\left[\begin{array}{lll}
\mathbf{e}_{\tau_{1}} \cdots & \mathbf{e}_{\tau_{L}}
\end{array}\right] \quad \mathbf{e}_{\tau_{i}} \\
& =\left[e^{-j w_{o} \tau_{i}} e^{-j w_{1} \tau_{i}} \ldots e^{-j w_{p} \tau_{i}}\right]^{T}
\end{aligned}
$$

The MV solution performs signals separation through the filter $\mathbf{w}$, as it is shown below, where the noise term $\widetilde{\mathbf{v}}(n)$ also accounts for the non-interesting paths:

$$
z(n)=\mathbf{w}^{H} \mathbf{y}(n)=a_{j}(n) \mathbf{w}^{H} \mathbf{G} \mathbf{e}_{\tau_{j}}+\mathbf{w}^{H} \tilde{\mathbf{v}}(n)
$$

The filter satisfies that $\mathbf{w}^{H} \mathbf{G e}_{\tau j}=1$, and an improved performance is achieved when $\mathbf{w}$ is chosen so as to maximize the output SNR or equivalently minimizing the noise output power:

$$
\begin{aligned}
\mathbf{w}= & \underset{\mathbf{w}^{*}}{\arg \min ^{H} E\left\{\mathbf{y}(n) \mathbf{y}(n)^{H}\right\} \mathbf{w}} \\
& \text { subject to } \quad \mathbf{w}^{H} \mathbf{G e}_{\tau_{j}}=1
\end{aligned}
$$

This minimization is performed using Lagrange multipliers, with $J$ being the cost function in Eq. (61):

$$
J=\mathbf{w}^{H} \mathbf{R}_{\mathbf{y}} \mathbf{w}+\lambda\left(\mathbf{w}^{H} \mathbf{G e}_{\tau_{j}}-1\right)
$$

And the achieved MV solutions for both the filter $\mathbf{w}$ and the spectral representations for delays as follow [62]:

$$
\mathbf{w}(\tau)=\frac{\mathbf{R}_{\mathbf{y}}^{-1} \mathbf{G e}_{\tau}}{\mathbf{e}_{\tau}^{H} \mathbf{G}^{H} \mathbf{R}_{\mathbf{y}}^{-1} \mathbf{G e}_{\tau}} \quad P(\tau)=\frac{1}{\mathbf{e}_{\tau}^{H} \mathbf{G}^{H} \mathbf{R}_{\mathbf{y}}^{-1} \mathbf{G e}_{\tau}}
$$

Note that one filter is found per each delay, and that the final power delay spectrum does not include the explicit expression of the filter. The determination of the timing for the first arrival from $P(\tau)$ requires the use of a threshold to avoid confusing the noise or the first side lobe with the true arrival [62].

\section{Results}

In the following section, several results will be shown for the CRBs for TOA in case of Rayleigh and Rice fading channels and also for the practical MV estimator in (62).

\subsection{Performance of asymptotic expressions}

Figure 2 compares the CRBs behavior for the estimated timing $k_{0}$ as a function of the temporal correlation for various values of the number of the observed channel

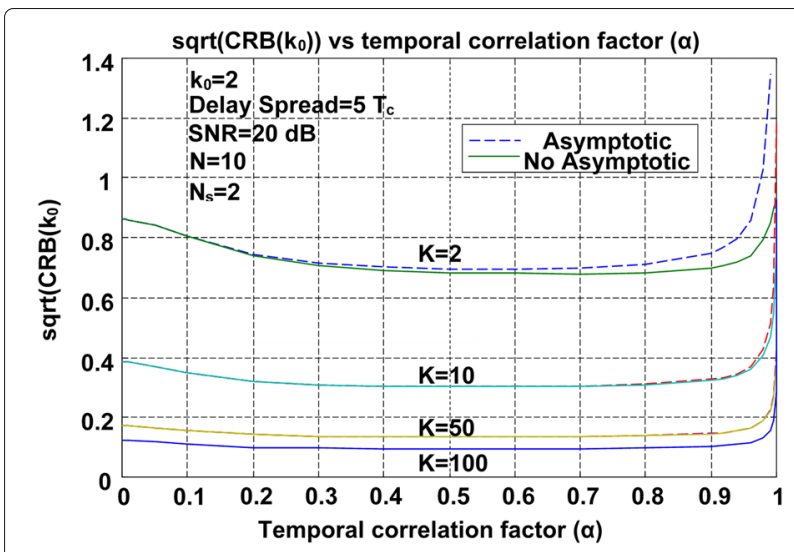

Fig. 2 CRB of the first arriving path $k_{0}$ in terms of the temporal correlation coefficient $\alpha$. Results for the square root of the CRB are displayed for different sizes $K$, of the record containing the channel vector estimates, for both the asymptotic (dashed) and no asymptotic (solid) expressions. SNR has been set to $20 \mathrm{~dB}$, delay spread to $5 T_{c}$, and for two sensors in the antenna array 
estimates, $K$, for the NLOS Rayleigh fading model in (35). Results were provided by using both the exact expressions in (36) and the expressions achieved with the use of the asymptotic eigenvalues from matrix $\mathbf{T}$ in (37) as described in the section "AF1.2" of Additional file 1. Asymptotic expressions for the first arrival timing $k_{0}$ fit very closely to exact ones for as few as 10 observations for this high SNR of $20 \mathrm{~dB}$ as it is shown in Fig. 2. However, higher values of $K$ were required when SNR were poor. For instance, when SNR was set to $0 \mathrm{~dB}$, instead of 10 observations, 50 observations were required to have a similar performance along the whole range of the temporal correlation. CRBs of the normalized coherence bandwidth $\lambda_{n}$ are more sensitive to the temporal correlation coefficient than their analog expressions for timing and 50 observations were required to achieve a good fitting for a SNR of $20 \mathrm{~dB}[35,36]$. For both parameters, the largest differences were achieved for a high temporal correlation coefficient very close to one where expected accuracies rapidly degrade.

Since timing is the parameter of interest for location purposes and based on the analysis exhibited in Fig. 1, it is expected that the average value of $K$ will be larger than 100 , very accurate results may be provided from the use of these asymptotic expressions, with the advantage of reducing computational burden since derivatives related to to the temporal correlation factor $\alpha$ were explicitly computed instead of using numerical methods.

\subsection{CRBs for timing and normalized coherence bandwidth for the NLOS Rayleigh fading model}

Figure 3 provides information about the behavior of the CRBs for the normalized coherence bandwidth $\lambda_{n}$ within $\Lambda_{\tau}$ in (20), in terms of SNR, and several values of $\alpha$ for the case of having different configurations at the antenna array. Specifically, the graphics at the top of Fig. 3 refer to the case of including two sensors at the antenna array while the graphics at the bottom of Fig. 3 refer to the case of including four. Note that the error bounds for this parameter are reduced for smaller temporal correlation coefficients. In fact, an improvement was registered when correlation shifted from 1 to 0 . Recall that this situation corresponds to subscribers changing from low to high speed respectively, or when channel estimates are achieved from more separated slots. CRB always diminished for higher SNRs, with some limiting floor value, which was significantly higher for the high temporal correlation case. This behavior also appears in CRBs for timing as shown in Figs. 4, 5, and 6. Note for example that the value of this error bound for the coherence bandwidth in Fig. 3 degraded from a value slightly better than $3 \%$ for the PCD source case $(\alpha=0.9)$ to around $9 \%$ for
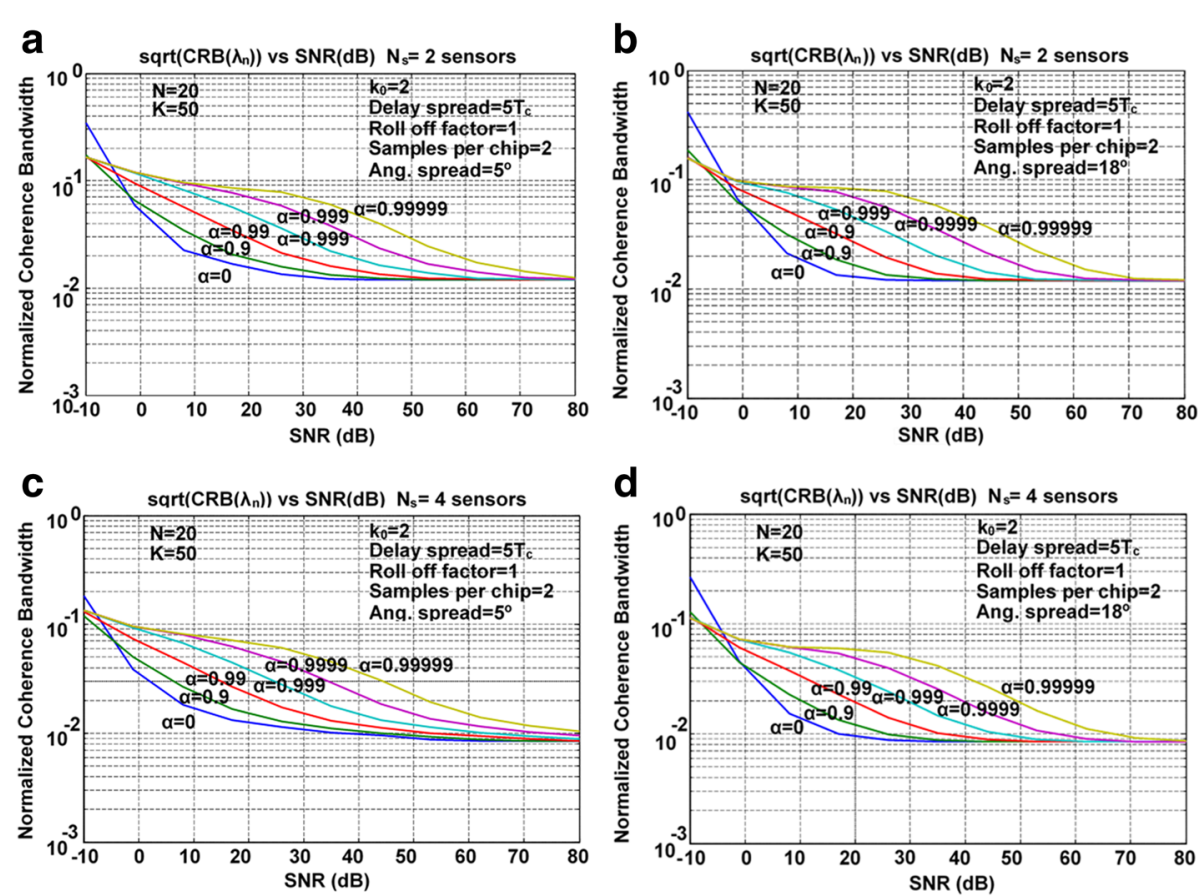

Fig. 3 CRB of the normalized coherence bandwidth $\lambda_{n}$ in terms of the SNR. Results are provided for different values of the temporal correlation $\alpha$, different values for the angular delay spread, and different values for the number of sensors $K$. The number of channel vector estimates $K$ has been set to 50 , the delay spread to $5 T_{c}$, and the roll-off factor to one. a Top left: angular spread set to $5^{\circ}$ and two sensors. b Top right: angular spread set to $18^{\circ}$ and two sensors. $\mathbf{c}$ Bottom left: angular spread set to $5^{\circ}$ and four sensors. $\mathbf{d}$ Bottom right: angular spread set to $18^{\circ}$ and four sensors 

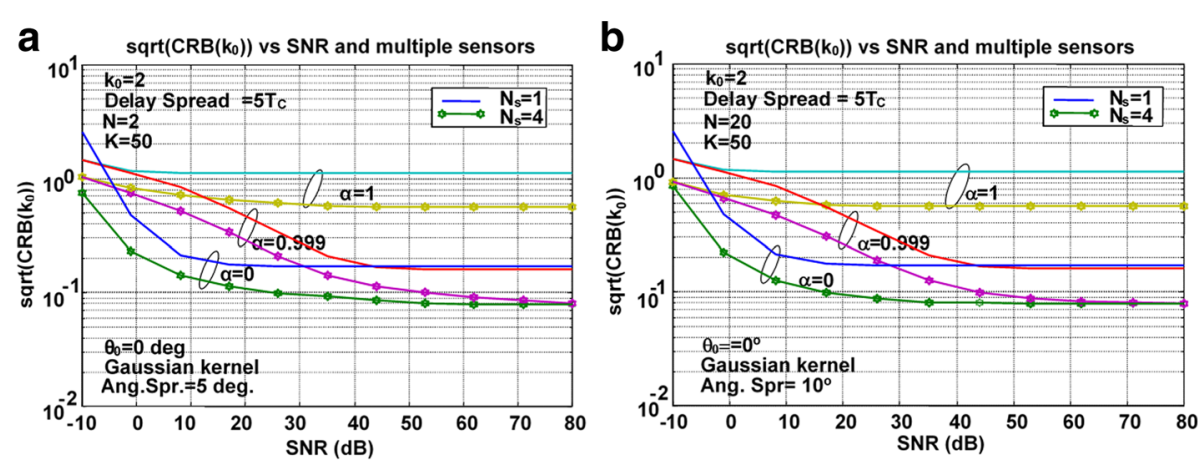

Fig. 4 CRB of the first arriving path $k_{0}$ for different values of the SNR. Results are provided for different values of the temporal correlation $\alpha$ and different angular spreads for the received signal. The number of sensors is 1 (solid) and 4 (solid bullet). Delay spread set to $5 T_{c}$, for 50 channel vector estimates. a Left: angular spread set to $5^{\circ}$. b Right: angular spread set to $10^{\circ}$

the FCD case when the SNR was set to $10 \mathrm{~dB}$, two sensors were used and the source angular spread corresponded to $5^{\circ}$. Furthermore, an improvement of the estimation lower than $25 \%$ was achieved when changing from two sensors to four. In addition, some slight reduction in the error bound was shown when angular spread increased from $5^{\circ}$ to $18^{\circ}$.

In addition, Fig. 4 shows the behavior of the CRB for the first arrival, when observations from signals received at multiple sensors were available. This figure compares the case of having just one sensor with the case where four sensors in $\lambda / 2$ were used in two environments with angular spreads of $5^{\circ}$ and $10^{\circ}$, which could be the case of UL measurements. These results showed that adding multiple antennas improved the accuracy of estimates significantly, but that angular spread did not significantly influence delay estimation. However, the improvement due to a higher angular spread resulted more important for lower delay spreads [35], and the best situation corresponded precisely to having completely uncorrelated sensors. In fact, the CRBs degraded as angular spread decreased. Differences were not really significant in relative terms, since computed errors were between 0.095 and 0.125 of the chip time, but they were more visible in range terms. For instance, range errors for a SNR of $15 \mathrm{~dB}$ were between 7.5 and $10 \mathrm{~m}$ for WCDMA and between 23 and $30 \mathrm{~m}$ for IS-95.

Furthermore, the inclusion of multiple sensors provided similar gains in timing accuracy, from moderate to high SNR, regardless of the value of the temporal coefficient. A gain factor of around two was achieved. In range terms, this means that error decreased from 16 to $8 \mathrm{~m}$ for ICD sources in a WCDMA system when a four sensor array was used instead of a single sensor.

Results from Figs. 1, 2, 3, and 4 above were achieved using a sampling equal to the chip rate, while Figs. 5 and 6 exhibit results for timing error bounds when sampling is faster than the chip rate. In particular, Fig. 5 shows that a marginal improvement in the timing error bound was performed when roll-off factor $\beta$ was modified from 0.5 to 1.0. For example, there was an improvement of just around $10 \%$ for a SNR of $15 \mathrm{~dB}$ for ICD sources. It accounts for less than a meter in range terms. However, due to the sampling being twice as fast, a gain of two was achieved
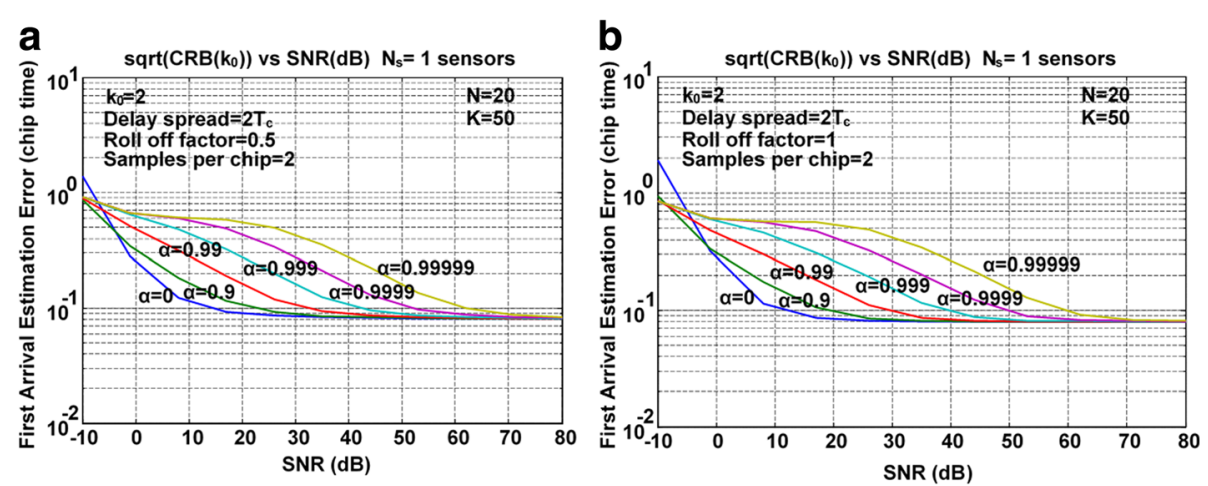

Fig. 5 CRB of the first arriving path $k_{0}$ for different values of the SNR. Results are provided for different values of the temporal correlation $\alpha$, delay spread set to $2 T_{c}$, and two different roll-off factors. One sensor and 50 channel vector estimates are available. The sampling rate set to twice the chip rate. a Left: set to 0.5 . b Right: $\beta$ set to 1.0 

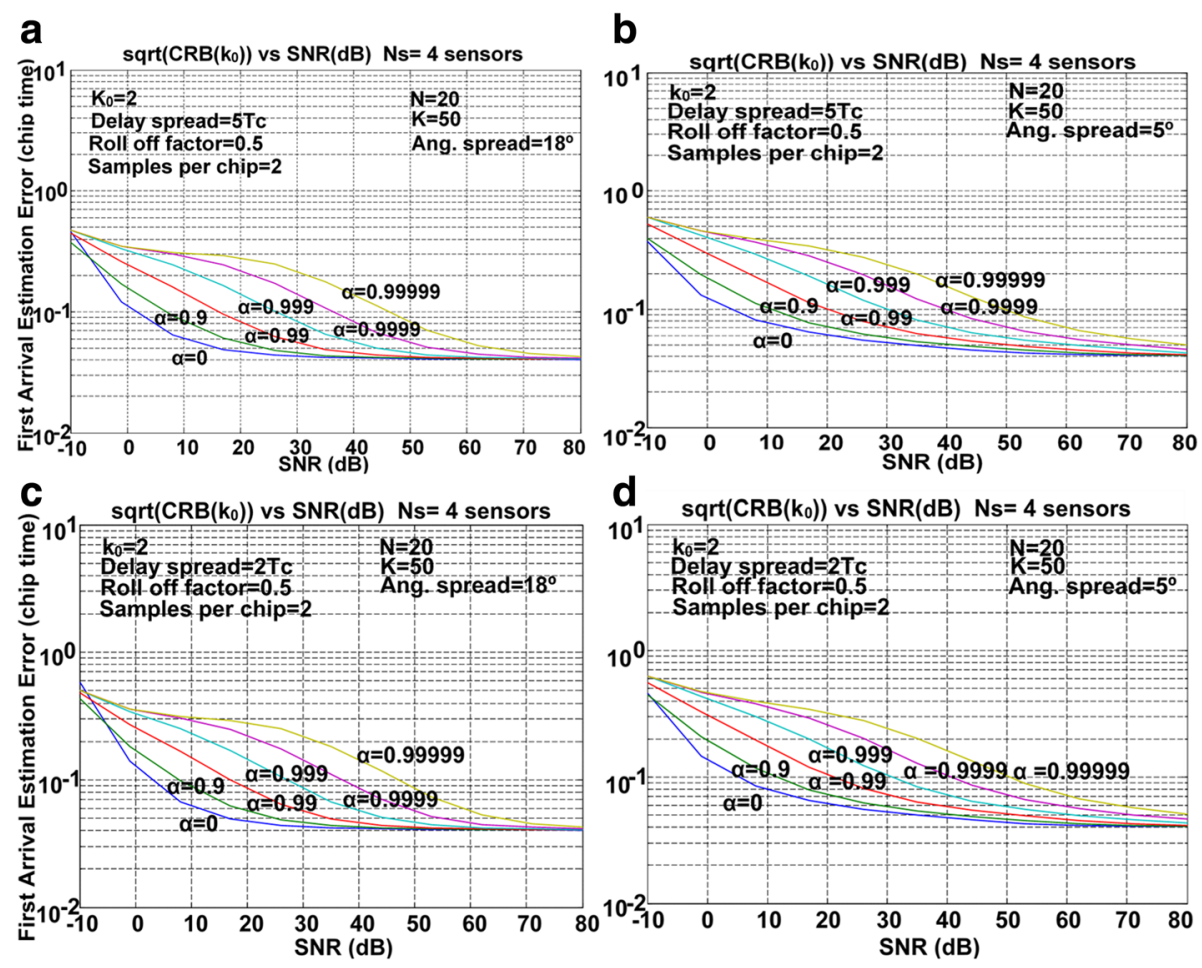

Fig. 6 CRB of the first arriving path $k_{0}$ for different values of the SNR. Results are provided for different values of the temporal correlation $\alpha$, different values of delay spread, and different values of angular spread. Four sensors and 50 channel vector estimates are available. The sampling rate set to twice the chip rate, and a roll-off factor of 0.5 . a Top left: delay spread set to $5 T_{c}$, angular spread set to $18^{\circ}$. $\mathbf{b}$ Top right: delay spread set to $5 T_{C}$, angular spread set to $5^{\circ}$. $\mathbf{c}$ Bottom left: delay spread set to $2 T_{c}$, angular set to $18^{\circ}$. d Bottom right: delay spread set to $2 T_{c}$, angular spread set to $5^{\circ}$

in the whole observed SNR range with independence of the temporal correlation factor $\alpha$. For example, the timing error for a SNR of $40 \mathrm{~dB}$ was reduced to around the half $\left(0.08 T_{c}\right)$ when the sampling rate was doubled as can be shown comparing Fig. 5 with results in [35].

Furthermore, Fig. 6 shows similar results in the timing error with independence of the angular and delay spread of the source. Again, major improvements were associated with a lower degree of correlation for the measures; however improvements related to angular and delay spreads were lower than a few meters in range terms. These improvements were performed for wider angular spreads, especially when SNR was low. This gain reduced for higher SNRs where errors tended to a minimum floor of of $0.04 T_{c}$, a corresponding range error of around $3 \mathrm{~m}$ in WCDMA.

Finally, Fig. 7 exhibits the timing error bound in terms of the number of estimates for different configurations of
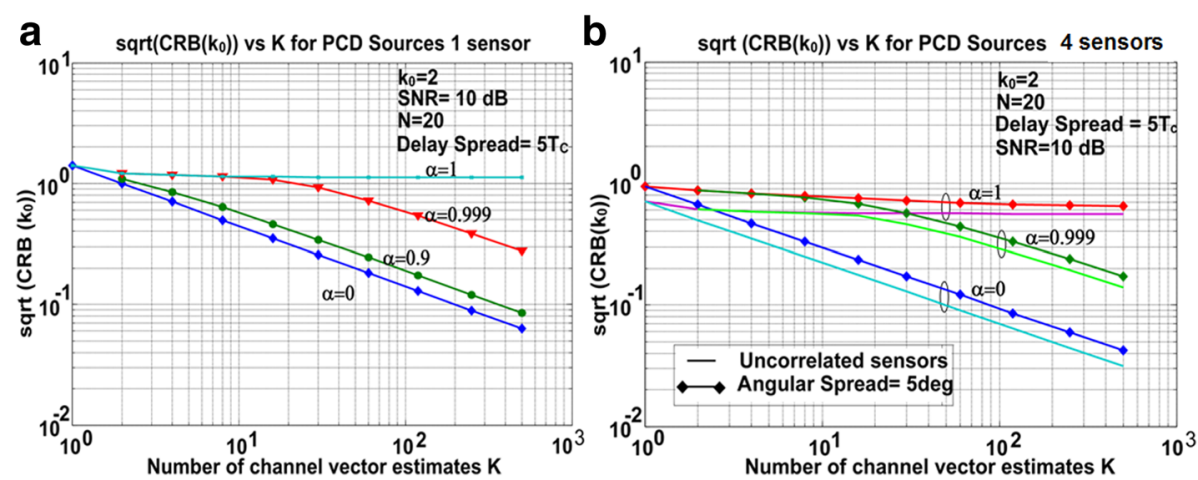

Fig. 7 CRB of $k_{0}$ as a function of the number of channel vector estimates $K$. Results are provided for different values of the temporal correlation coefficient $\alpha, \mathrm{SNR}=10 \mathrm{~dB}$, and a delay spread of $5 T_{c}$. a Left: one sensor. b Right: four sensors and two possible scenarios: uncorrelated sensors case and a low angular spread case (angular spread of $5^{\circ}$ ) 

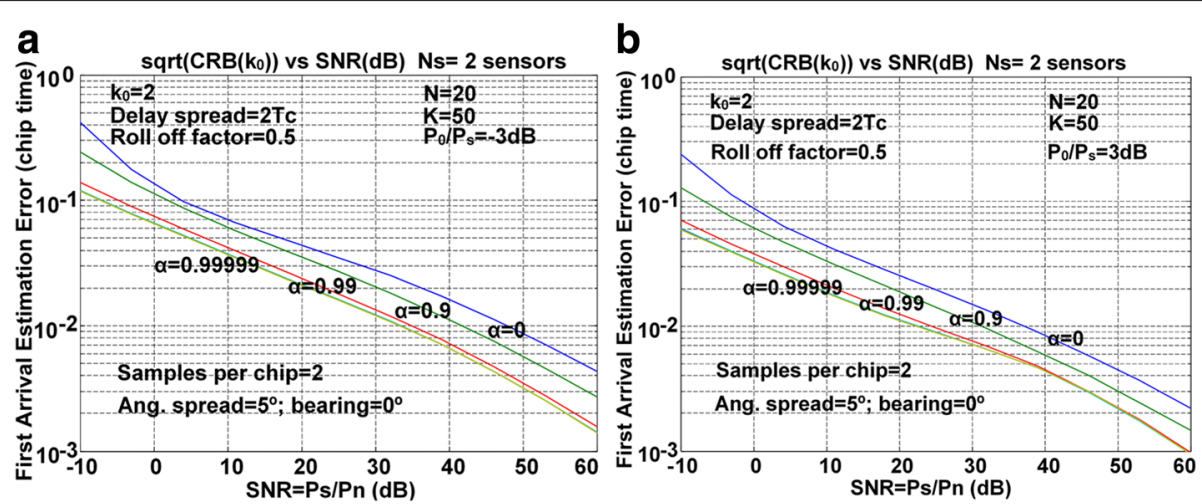

Fig. 8 CRB of the first arriving path $k_{0}$ as a function of dispersed SNR. Results are provided for different values of the temporal correlation coefficient $\alpha$ and for different values of the LOS component power. Two sensors and 50 channel vector estimates are available. The sampling rate set to twice the chip rate and a roll-off factor of 0.5. Delay spread set to $2 T_{c}$ and angular spread set to $5^{\circ}$. Bearing direction is the broadside. $\mathbf{a}$ Left: LOS power set $3 \mathrm{~dB}$ lower than the dispersed signal power. b Right: LOS power set $3 \mathrm{~dB}$ higher than the dispersed signal power

the antenna array and several temporal correlation factors among the observed estimates. This figure shows that the first arrival timing estimation error bound improves as the number of channel vector estimates $K$ increases. In the case of highly temporally correlated channel estimates, results showed the difficulty to reduce the error bounds, even for a large number of observations (high values of $K$ ) or when multiple antennas were used. As an example, note that in Fig. 7 left that error reduced to the third from 4.8 to $1.2 \mathrm{~m}$ in range terms, in case of $\alpha=0.9$; and to just half from 9.6 to $4.8 \mathrm{~m}$ when $\alpha=0.999$ and $K$ passed from 10 to 100 . The influence of the angular spread is another factor to be considered: spatial uncorrelated sensors allowed a better estimation of the timing, but improvement considered in range terms was more important in cases of having highly temporally correlated estimates since in this case, for example, it accounted for around $8 \mathrm{~m}$ for $K=100$ and just around of $1.5 \mathrm{~m}$ when channel estimates were temporally uncorrelated.
Interpretation of results should be made very carefully when the number of observations is analyzed in relation with the time correlation factor $\alpha$, since channel estimates achieved from two consecutive slots will exhibit a higher temporal correlation factor compared to those performed using much more separated slots; and also considering that the coherence time for delays reduces for higher mobile speeds and therefore limits the availability of new estimates.

On the other hand, and from the perspective of the computation of CRBs, the use of uncorrelated sensors implies a reduction in complexity since the spatial correlation in (31) disappears as a nuisance parameter and computation therefore becomes simpler and faster.

\subsection{CRBs for timing and normalized coherence bandwidth for the LOS Rice fading model}

This section shows behavior of the error bounds for the timing $k_{0}$ and the normalized coherence bandwidth $\lambda_{n}$ in
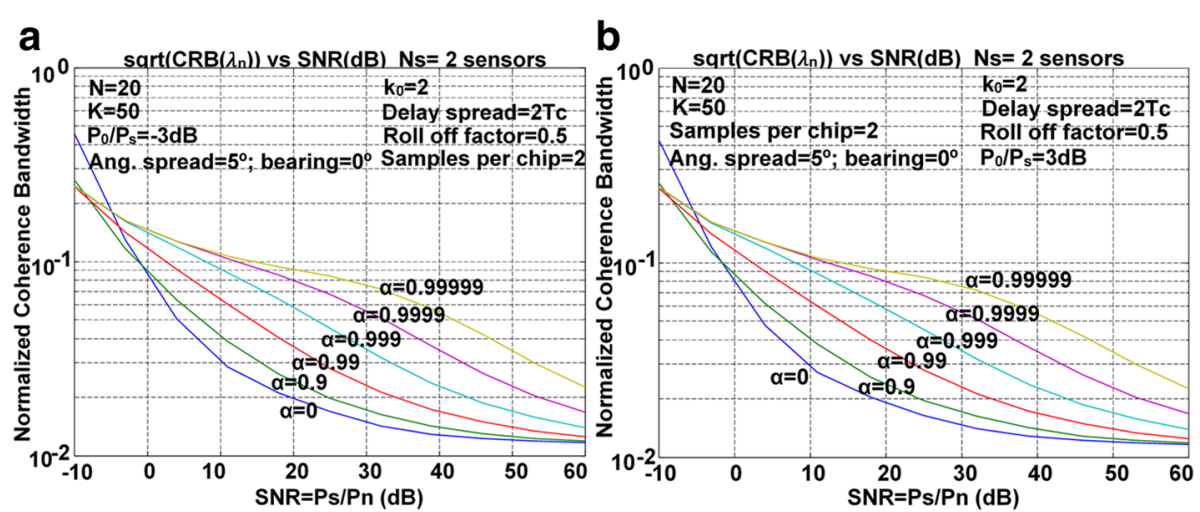

Fig. 9 CRB of the normalized coherence bandwidth $\lambda_{n}$ in terms of the dispersed SNR. Results are provided for different values of the temporal correlation coefficient $\lambda$, and for different values of the LOS component power. Two sensors and 50 channel vector estimates are available. The sampling rate set to twice the chip rate and a roll-off factor of 0.5 . Delay spread set to $2 T_{c}$ and angular spread set to $5^{\circ}$. Bearing direction is the broadside. a Left: LOS power set $3 \mathrm{~dB}$ lower than the dispersed signal power. b Right: LOS power set $3 \mathrm{~dB}$ higher than the dispersed signal power 
case of the LOS Rice model in (50). Particularly, Figs. 8 and 9 exhibit exhibit these bounds as a function of the dispersed SNR for two different values of the average LOS power: $3 \mathrm{~dB}$ above and $3 \mathrm{~dB}$ below the dispersed signal. First of all, note that the timing error reduced for higher temporally correlated environments when a LOS component was present. This behavior was precisely the opposite of what was registered for a NLOS condition. Furthermore, timing bounds computed for this LOS model were lower than those expected for the NLOS condition, and they reduced as LOS power increased. Improvement achieved for higher temporal correlation is almost negligible for temporal correlation factors higher than 0.99. For example, note that the timing error for a SNR of $15 \mathrm{~dB}$ and an ICD source $(\alpha=0)$ with a LOS power $3 \mathrm{~dB}$ below the dispersed signal power when two sensors were used corresponded to $5.5 \times 10^{-2} \mathbf{T}_{c}$. This bound reduced to $4.8 \times 10^{-2} \mathbf{T}_{c}$ for a PCD source $(\alpha=0.9)$, and to $3.0 \times 10^{-2} \mathbf{T}_{c}$ for a FCD source $(\alpha=0.99999)$. When the LOS power increased to $3 \mathrm{~dB}$ higher than the dispersed signal power, the bound reduced from $3.2 \times 10^{-2}$ $\mathbf{T}_{c}$ to $2.5 \times 10^{-2} \mathbf{T}_{c}$ and finally to $1.5 \times 10^{-2} \mathbf{T}_{c}$ for ICD $(\alpha=0), \operatorname{PCD}(\alpha=0.9)$, and FCD $(\alpha=0.99999)$ cases, respectively. In range terms, it means that distance error went from around $4.4 \mathrm{~m}$ (ICD) to $2.4 \mathrm{~m}$ (FCD) for the first case and from 2.6 to $1.2 \mathrm{~m}$ for the latter. Another interesting observation is the fact that timing error reduced without bound when SNR increased for this LOS model. This indicates that timing accuracy would be theoretically limited just by SNR in cases of a dominant LOS condition.

These results perhaps seem to be too optimistic, but they are consistent with the model structure that supposes the LOS signal is perfectly characterized. In fact, if the signal we are looking for is practically deterministic, which it is especially true in high SNR conditions; it is possible to estimate the timing with a very high accuracy.

On the other hand, the bounds for the normalized coherence bandwidth $\lambda_{n}$ seemed not to be disturbed for a change in the LOS power level, and the tendency in relationship to the temporal correlation coefficient remained consistent as in the NLOS model. For example, note from Fig. 9 that the error bounds were somewhat higher than those expected from the NLOS model, but they were also bound limited when SNR increased. In this case, the minimum error bound achievable was around $1.2 \%$. This slight degradation exhibited in CRB for the LOS model is derived from the fact that the vector of unknown parameters included a new parameter to estimate [26], and due to the fact that this new LOS parameter did not disturb

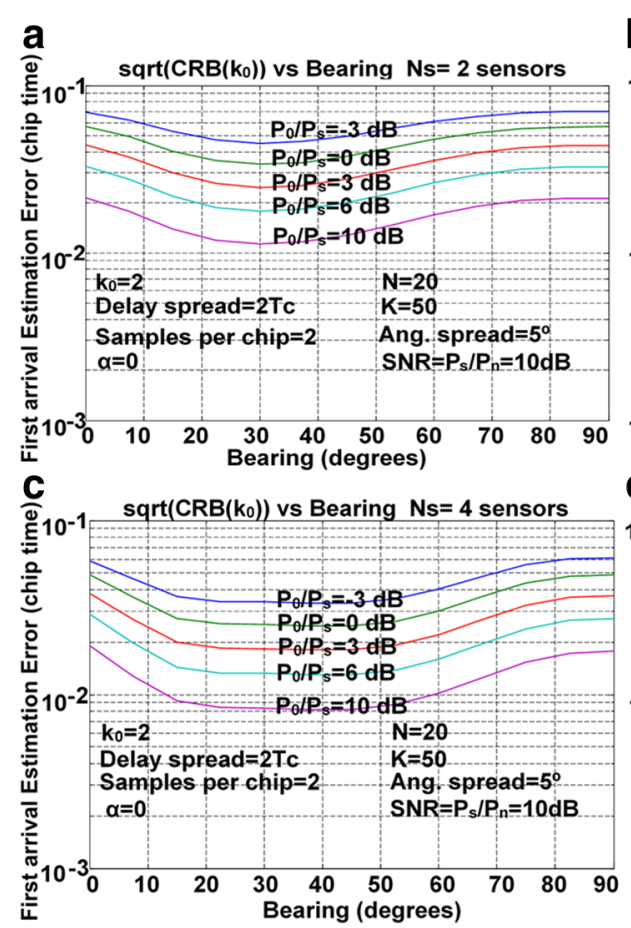

b

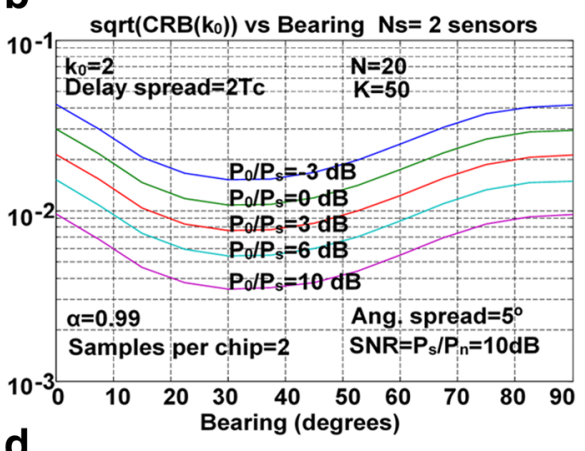

d

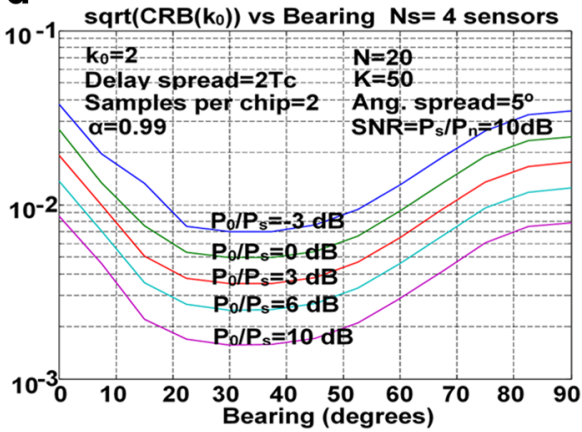

Fig. $10 \mathrm{CRB}$ of the first arriving path $k_{0}$ as a function of the signal bearing. Results are provided for different values of the power level of the LOS component and various values of the temporal correlation coefficient $\alpha$. 50 channel vector estimates are available. The sampling rate set to twice the chip rate, and a roll-off factor of 0.5. Delay spread set to $2 T_{c}$, angular spread set to $5^{\circ}$ and the dispersed SNR set to $10 \mathrm{~dB}$. a Top left: two sensors-ICD source $(\alpha=0)$. b Top right: two sensors-highly temporal correlated source $(\alpha=0.99)$. c Bottom left: four sensors-ICD source $(\alpha=0)$; $\mathbf{d}$ Bottom right: four sensors-highly temporal correlated source $(\alpha=0.99)$ 

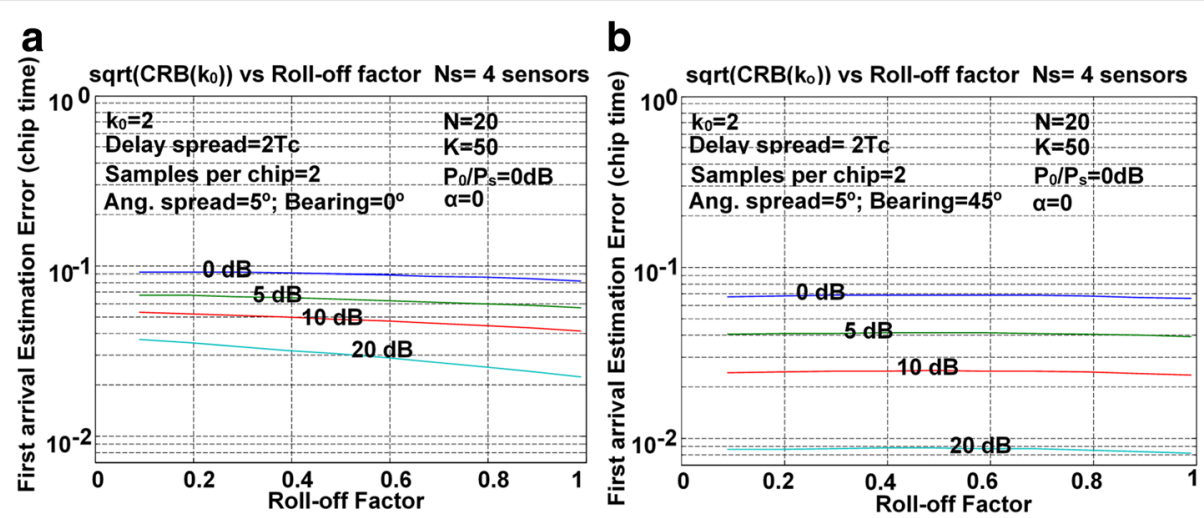

Fig. $11 \mathrm{CRB}$ of $k_{0}$ as a function of the roll-off factor of the shaping pulse. Results are provided for different values of the dispersed SNR when the LOS power level is $0 \mathrm{~dB}$ higher than the dispersed signal and an ICD source $(\alpha=0) .50$ channel vector estimates are available. The sampling rate set to twice the chip rate, delay spread set to $2 T_{c}$, angular spread set to $5^{\circ}$, and four sensors. $\mathbf{a}$ Left: bearing $=0^{\circ}$. $\mathbf{b}$ Right: bearing $=45^{\circ}$

temporal dispersion statistics in the model. However, it is important to point out, that a LOS condition is associated with a less dispersed signal both temporally and spatially $[15,17,42]$, and this fact has to be considered in the analysis to extract proper conclusions from these results. Furthermore, from a positioning viewpoint, timing is the most relevant parameter, and the coherence bandwidth can be considered a nuisance parameter. Nevertheless, from a systemic perspective this parameter could provide some additional information about the quality of the measure [42].

Figures 10 and 11 relate space-time diversity with the timing error bounds for our Rice fading model. Particularly, Fig. 10 shows the behavior of this bound with the mean direction of arrival of the received signal for different power values of the LOS component when the dispersed SNR was set to $10 \mathrm{~dB}$. The impact of temporal correlation is also assessed by comparing results performed for ICD sources on the left of the figure with those achieved for PCD sources on the right side. Furthermore, the gain introduced for the use of a larger number of sensors is also exhibited by comparing graphics at the top (two sensors) with those at the bottom (four sensors).

First of all, it is interesting to note that the timing error reached a minimum for values close to $30^{\circ}$ and that this improvement became more important in relative terms for higher levels of the LOS component and for more temporally correlated signals, since in these cases, the possibly became almost deterministic and was easier to be discriminated from noise. Furthermore, for this LOS model, a better gain was performed from the introduction of new sensors for the case of PCD sources. For example, a gain factor of around 1.35 was achieved when passing from two sensors to four in case of ICD sources and this factor increased to around two for PCD sources $(\alpha=0.99)$. Bearing also impacted the timing error performance. A higher gain was found when the mean signal bearing was around $35^{\circ}$, and the range of the improvement region widened around this bearing when more sensors were added and a higher LOS power was available. This gain decayed when the LOS path weakened and the Rice propagation turned into Rayleigh. Gains associated bearing reduced the timing error below half for high power LOS signals, and these errors were reduced around $45 \%$ when the LOS power changed from -3 to $3 \mathrm{~dB}$ over the disperse component when four sensors were used.

On the other hand, Fig. 11 relates the CRB for the timing error with the roll-off factor of the shaping pulse, the SNR, and also with the signal bearing. Results in this figure demonstrated that the timing error bound improved for a higher roll-off factor when the signal arrived directly from the broadside (Fig. 11-left), especially for high signal to noise ratios. This enhancement is possibly related with the sharper form of the first arrival related to the increase in the bandwidth. However, the gain with roll-off factor was negligible when bearing changed to $45^{\circ}$ (Fig. 11-right) due the better array performance for this bearing.

For example, note from the graphics that as the timing error reduced from $3 \times 10^{-2} T_{c}$ for a roll-off factor of 0.5 to around $2 \times 10^{-2} T_{c}$ for a roll-off factor of 1.0 when four sensors were used, the dispersed SNR was set to $20 \mathrm{~dB}$, and signal arrived directly from the broadside (Fig. 11-left). On the other hand, when direction of arrival changed to $45^{\circ}$, timing error kept very close to $0.85 \times 10^{-2} T_{c}$ with independence of the roll-off factor for the same signal conditions (Fig. 11-right). Of course, lower errors were achieved when a larger number of sensors were used. The behavior described by these results is very reasonable since modifying the pulse shape to a higher roll-off implies the availability of a higher bandwidth, and therefore the reduction of the side lobes. Therefore, it helps to reduce the probability of missing the first arrival during the estimation stage. Furthermore, the array geometry responds to bearing, and it can help 
MV-Estimation: $\quad \mathrm{N}_{\mathrm{s}}=4 ; \mathrm{K}=75$ channel estimates; Temporal correlation $=0 ; 2 \mathrm{~s} / \mathrm{symb} ; \mathrm{SNR}=10 \mathrm{~dB}$

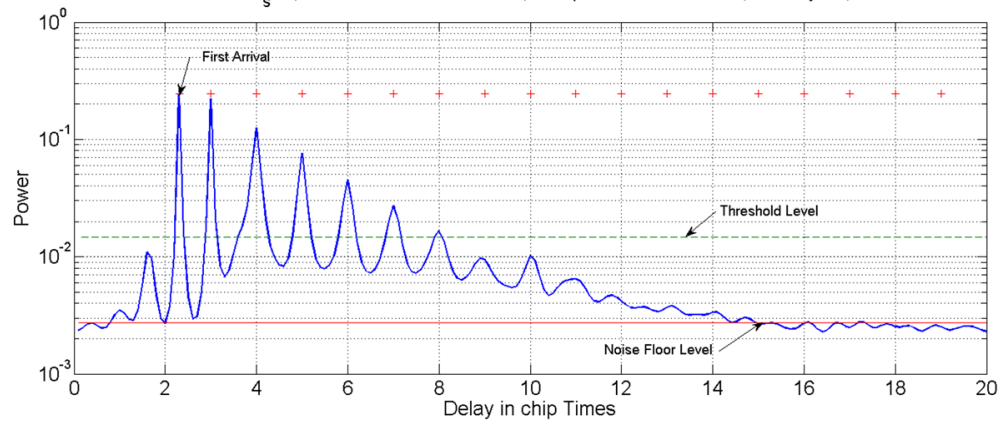

Fig. 12 Minimum variance spectrum for timing estimation. This result is provided for a SNR of $10 \mathrm{~dB}$, an ICD source $(\alpha=0), 75$ channel vector estimates available; the sampling rate set to twice the chip rate, delay spread set to $2 T_{c}$, angular spread set to $5^{\circ}$, and four sensors

to discriminate the LOS component from the dispersed signal.

\subsection{Practical estimators and CRBs}

Figure 12 exhibits the TOA-MV power spectrum computed using (62) for a NLOS signal model as described by (17) and (19) for an ICD source, when SNR=10 dB, $K=75$ channel estimates, and $N_{s}=4$ sensors for a sampling of twice the chip rate. The threshold has been computed properly [62] over the noise floor to avoid an early detection due to the first side lobe. On the other hand, Fig. 13 shows the root mean square error (RMSE) for the first timing as a function of SNR and the temporal correlation coefficient, computed over 2,500 realizations for two different configurations by using the MV approach. Both groups of results were computed for $K=50$ estimates, $N=20$ chip times, an angular spread of $5^{\circ}$, and a delay spread of $2 T_{c}$, and they are compared with results achieved for the CRBs. Results on the left correspond to a configuration with $N_{s}=1$ sensor and a sampling of twice the chip rate, while results to the right corresponds to $N_{s}=4$ sensors and sampling performed to the chip rate. Estimated errors exhibited for the case of an ICD source are slightly higher than those provided for the corresponding bounds, especially when just one sensor is available. However, higher errors have been measured when the temporal correlation increases especially when SNR is low. When SNR increases the error decays to the minimum as was expected. When $N_{s}=4$, errors also diminish as before, but although error tries to attain the CRB at high values of SNR, the minimum error is finally higher than the expected by the CRBs. These results provide evidence of the strengths and weakness from both the CRBs and the practical estimator at hand. In essence, the proper behavior of the MV-timing estimator has been verified by the bounds for the best possible scenario: an ICD source. However, the impossibility of this method to attain the bounds in more aggressive scenarios reveals that the current formulation of this algorithm is possibly not taking advantage of all the information provided by the temporal and spatial diversity and therefore it is not the MVU estimator. On the other hand, these discordances
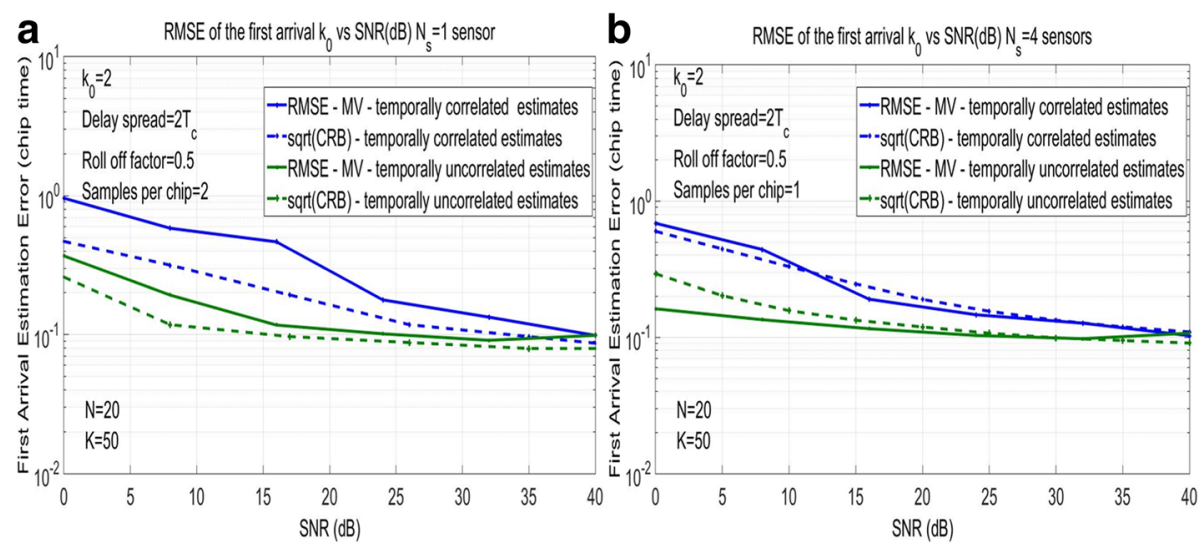

Fig. 13 Root mean square error of the first arriving path $k_{0}$ for different values of the SNR. Results are provided for different values of the temporal correlation $\alpha$, and different configurations of the antenna array, a delay spread of $2 T_{c}, 50$ channel vector estimates available, and an angular spread of $5^{\circ}$. The roll-off factor set to 0.5 . $\mathbf{a}$ Left: one sensor and the sampling rate set to twice the chip rate. $\mathbf{b}$ Right: one sensor and the sampling rate set to the chip rate 
remind us that the CRB is an optimistic model for any unbiased estimator that alerts us about the inherent difficulties of performing the estimation. In this case, the bounds are a warning about addressing the temporal correlation of the estimates in order to get the best results out of the method. High correlated estimates result in ill-conditioned matrices degrading the behavior of this practical estimator.

\section{Conclusions}

This paper describes the use of the CRBs to study the impact of various factors involved in signal TOA estimation for a mobile scenario modeled by a space-time dispersive channel for both Rayleigh and Rice fading propagation situations, and therefore, it explores the difficulties and opportunities associated with timing estimation in LOS and NLOS environments.

1. In particular, our model makes a contribution by taking into account the spatial and temporal correlation among channel estimates, and the impact of the roll-off factor of the shaping pulse, in addition to the number of sensors and the number of estimates that are typical from other approaches. It also includes an exponential dispersion for delays which is characteristic of mobile channels instead of just a few paths as in prior approaches. Furthermore, this paper also includes some asymptotic expressions for certain interesting cases related to with high speed and low speed subscribers.

2. Due to the close relationship between timing and positioning, this model does contribute to insight not only to the TOA estimation but also to its impact on positioning.

From results in Section 3, the following conclusions can also be derived:

1. Estimation errors for the timing and the normalized coherence bandwidth decrease when the SNR increases; but this improvement is highly conditional depending on the propagation scenario and the type of source. In the case of NLOS Rayleigh propagation, these estimation errors degrade rapidly when passing from PCD sources to FCD sources, reaching a limit floor at high SNRs, so a higher SNR does not force a lower error. On the other hand, in case of LOS Rice propagation, the larger improvement is achieved when passing from an ICD source to a PCD source, and timing accuracy improves practically without bound for higher SNRs.

2. The bounds for the normalized coherence bandwidth $\lambda_{n}$ seem not to be disturbed by a change in the LOS power level; results remain as in the NLOS model.

3. Estimation errors, for the timing and the normalized coherence bandwidth, also decrease when the number of observations increases, but again this reduction is very conditioned on the propagation scenario and the kind of source: ICD, PCD, or FCD. In the case of NLOS, a larger record of observations is required to keep the accuracy for higher temporal correlations among channel estimates; however in the case of a LOS scenario, an uncorrelated dispersed signal component implies a random perturbation that degrades the accuracy on the signal of interest.

4. The use of multiple antennas introduces not just new observations but also diversity, and therefore it helps to improve accuracy. However, the impact of these improvements is associated with temporal and spatial coherence of the scattered signal. For the NLOS condition, inclusion of multiple sensors provides similar gains in timing accuracy, from moderate to high SNR, regardless of the value of the temporal coefficient. A gain factor of around two is achieved when passing from one sensor to four, confirming the observations in [31]. However, in LOS condition, this gain almost doubles in the case of highly PCD sources, and the bearing also impacts the timing error performance. This gain decays when the LOS path weakens and the Rice propagation turns into Rayleigh. Improvements are always obtained when an antenna array is used instead of a single sensor, and certain improvements of around $20 \%$ are also achieved when passing from a narrow spread source to a spatially well-scattered signal.

5. Under a non line of sight (NLOS) condition, the roll off factor has negligible effect on error bounds, while under LOS condition, a higher roll-off factor helps to improve the bound for the timing error, possibly due to the sharper form of the first arrival in this case, related to the increase in the bandwidth.

6. CRBs provide a useful and optimistic insight about the estimation problem that may help to achieve practical and efficient estimators.

Finally, the following recommendations should be taken into account:

1. In spite of our CRB model providing valuable information about the timing estimation error, careful attention is required to extrapolate these results to the mobile subscriber positioning issue, due to the different nature of Rayleigh and Rice propagation models. For example in obstructed environments, the shadowing may lead to important delay spreads, while in LOS condition, low delays are expected. In addition, some obstructed scenarios may lead to signal clustering, and if that is the case, even with the first arrival being accurately estimated, the positioning could be biased. Fortunately, there are 
some methods to identify these scenarios [33, 40, 63] and to reduce the harmful effects of this NLOS condition.

2. Errors from the measures translate directly into range errors for positioning based on TOA, and these certainly degrade the subscriber's positioning. However, it would be inappropriate to think about the range errors as final positioning errors. Positioning is a more complex procedure that involves the acquisition from signals transmitted and received from different parts of the network, and therefore it is also dependent on the problem geometry. However, the use of larger data records dramatically reduces the positioning error, and therefore it is very important to determine the coherence time of delays and angles to take advantage of this situation. In fact, positioning accuracy is very sensitive to the subscriber mobility, being the highest error associated with static equipment in NLOS condition, due to the impossibility to taking advantage of temporal diversity.

\section{Additional file}

Additional file 1: AF1.1 CRBs for the NLOS Rayleigh Fading Model when sampling is performed at the chip rate. AF1.2 Asymptotic Expressions for Delay Estimates and a PCD Source. AF1.3 CRB's for Delay Estimates in case of Fully Coherent Dispersed Sources. (PDF 211 kb)

\section{Abbreviations}

BB: Barankin bound; BCRB: Bayesian CRB; BS: Base station; CRB: Cramer-Rao bound; DOA: Direction of arrival; FCD: Fully coherent dispersed; FIM: Fisher information matrix; GNSS: Global navigation satellite system; ICD: Incoherent dispersed; KF: Kalman filter; LOS: Line of sight; MCRB: Modified CRB; ML: Maximum likelihood; MS: Mobile station; MSE: Mean squares error; MV: Minimum variance; MVU: Minimum variance unbiased; MZZB: Modified ZZB; NLOS: Non line of sight; OTDO: Observed time differences of arrival; PAS: Power angular spectrum; PCD: Partially coherent dispersed; PCF: Position computing function; RMSE: Root mean square error; SNR: Signal to noise ratio; SS: Signal strength; TDOA: Time differences of arrival; TOA: Time of arrival; UWB: Ultra wide band; WCDMA: Wideband code division multiple access; ZZB: Ziv-Zakai bound

\section{Acknowledgements}

The authors thank Andy Espinosa Gutiérrez for his help in the Latex edition of this paper, David Barmettler for his kind review of the English text, and finally the anonymous reviewers whose comments helped to improve the quality of this document.

\section{Funding}

This work was carried out in the framework of the EC-funded project Saturn IST-1999-10322 and FUNDACYT subvention 980349 from Ecuador.

\section{Authors' contributions}

In addition to the original idea, a continuous support and feedback were performed by Dr. JV along the whole development of this work. Mathematical developments, algorithms implementation, results depuration, and article's writing were developed by the first author. Both authors read and approved the final manuscript.

\section{Competing interests}

The authors declare that they have no competing interests.

\section{Publisher's Note}

Springer Nature remains neutral with regard to jurisdictional claims in published maps and institutional affiliations.

\section{Author details}

${ }^{1}$ Universidad San Francisco de Quito, Diego de Robles y Pampite, Quito, Ecuador. ${ }^{2}$ Universitat Politècnica de Catalunya, Campus Nord, c/ Jordi Girona 1-3, Barcelona, Spain.

Received: 10 June 2016 Accepted: 21 February 2018

Published online: 16 March 2018

\section{References}

1. Technical Specification Group Services and System Aspects; Location Services (LCS); Service Description; Stage 1 (Release 9) - 3GPP TS 22.071 V9.1.0 (2010-09). (ETSI 3RD Generation Partnership Project, 2010). http://www.qtc.jp/3GPP/Specs/22071-910.pdf. Accessed 9 Jan 2016

2. J Johansson, WA Hapsari, S Kelley, G Bodog, Minimization of Drive Tests in 3GPP Release 11. IEEE Commun. Mag. 50(11), 36-43 (2012). https://doi.org/ 10.1109/MCOM.2012.6353680

3. M Abo-Zahhad, SM Ahmed, M Mourad, Future location prediction of mobile subscriber over mobile network using Intra Cell Movement pattern algorithm. IEEE 1st Int. Conf. Communic. Signal Proc. Appl. 1-6 (2013). https://doi.org/10.1109/ICCSPA.2013.6487272

4. R Barnes, B Rosen, 911 for the 21st century. IEEE Spectr. 51(4), 58-64 (2014). https://doi.org/10.1109/MSPEC.2014.6776307

5. C-M Huang, S-C Lu, DEH: A ubiquitous Heritage Exploring System using the LBS Mechanism. IEEE Int. Conf. Netw.-Based Inf. Syst. 310-317 (2011). https://doi.org/10.1109/NBiS.2011.54

6. M Driusso, M Comisso, F Babich, C Marshall, Performance analysis of time of arrival estimation on OFDM signals. IEEE Signal Process. Lett. 22(7), 983-987 (2015). https://doi.org/10.1109/LSP.2014.2378994

7. J Huang, P Wang, Q Wan, CRLBs for WSNs localization in NLOS environment.EURASIP. J. Wirel. Commun. Netw. 2011, 16 (2011). https://doi.org/10.1186/1687-1499-2011-16

8. L Cong, W Zhuang, Hybrid TDOA/AOA mobile user location for wideband CDMA cellular systems. IEEE Trans. Wirel. Commun. 1(3), 439-447 (2002). https://doi.org/10.1109/TWC.2002.800542

9. K Pahlavan, X Li, Indoor Geolocation Science and Technology. IEEE Commun. Mag. 40(2), 112-118 (2002). https://doi.org/10.1109/35.983917

10. A Catovic, Z Sahinoglu, The Cramer-Rao bounds of hybrid TOA/RSS and TDOA/RSS location estimation schemes. IEEE Commun. Lett. 8(10), 626-628 (2004). https://doi.org/10.1109/LCOMM.2004.835319

11. Y Wang, G Leus, Reference-free time-based localization for an asynchronous target. EURASIP J. Adv. Signal Process. 2012, 19 (2012). https://doi.org/10.1186/1687-6180-2012-19

12. Y Wang, Linear least squares localization in sensor networks. EURASIP J. Wirel. Commun. Netw. 2015, 51 (2015). https://doi.org/10.1186/s13638015-0298-1

13. S Ahonen, P Eskelinen, Mobile terminal location for UMTS. IEEE Aerosp. Electron. Syst. Mag. 18(2), 23-27 (2003). https://doi.org/10.1109/MAES. 2003.1183866

14. G Fuks, J Goldberg, H Messer, Bearing estimation in a Ricean channel_-part I: inherent accuracy limitations. IEEE Trans. Signal Process. 49(5), 925-937 (2001). https://doi.org/10.1109/78.917797

15. H Asplund, AA Glazunov, AF Molisch, KI Pedersen, M Steinbauer, The COST 259 directional channel model — part II: macrocells. IEEE Trans. Wirel. Commun. 5(12), 3434-3450 (2006). https://doi.org/10.1109/TWC.2006.256967

16. P Lusina, F Kohandani, SM Ali, Antenna parameter effects on spatial channel models. Inst. Eng. Technol. Commun. 3(9), 1463-1472 (2009). https://doi.org/10.1049/iet-com.2008.0414

17. C Gentile, S Martínez, A Kik, A comprehensive spatial-temporal channel propagation model for the Ultrawideband Spectrum 2-8 GHz. IEEE Trans. Antennas Propag. 58(6), 2069-2077 (2010). https://doi.org/10.1109/TAP. 2010.2046834

18. M Bengtsson, B Ottersten, Low-complexity estimators for distributed sources. IEEE Trans. Signal Process. 48(8), 2185-2194 (2000). https://doi. org/10.1109/78.851999

19. O Besson, P Stoica, Decoupled estimation of DOA and angular spread for a spatially distributed source. IEEE Trans. Signal Process. 48(7), 1872-1882 (2000). https://doi.org/10.1109/78.847774

20. S Valaee, B Champagne, P Kabal, Parametric localization of distributed sources. IEEE Trans. Signal Process. 43(9), 2144-2153 (1995). https://doi. org/10.1109/78.414777 
21. GC Raleigh, T Boros, Joint space-time parameter estimation for wireless communication channels. IEEE Trans. Signal Process. 46(5), 1333-1343 (1998). https://doi.org/10.1109/78.668795

22. M Wax, A Leshem, Joint estimation of time-delays and direction of arrival of multiple reflections of a known signal. IEEE Trans. Signal Process. 45(10), 2477-2484 (1997). https://doi.org/10.1109/78.640713

23. J Lee, CH Lee, J Chun, JH Lee, Joint estimation of space-time distributed signal parameters. IEEE Conf. Veh. Technol. 2, 822-828 (2000). https://doi. org/10.1109/VETECF.2000.887118

24. TMenni, E Chaumette, P Larzabal, Reparameterization and constraints for CRB: duality and a major inequality for system analysis and design in the asymptotic region. IEEE Int. Conf. Acoust. Speech Signal Process. 3545-3548 (2012). https://doi.org/10.1109/ICASSP.2012.6288682

25. A Emmanuele, M Luise, Fundamental limits in signal time-of-arrival estimation in AWGN and multipath scenarios with application to next-generation GNSS. IEEE ESA Workshop Satell. Navig. Technol. Eur. Workshop GNSS Signals Signal Process, 1-7 (2010). https://doi.org/10. 1109/NAVITEC.2010.5708049

26. SM Kay, Fundamentals of statistical signal processing - estimation theory, 16th edn. (Prentice Hall, New Jersey, 1993), pp. 27-63

27. S-M Omar, D Slock, O Bazzi, Recent insights in the Bayesian and deterministic CRB for blind SIMO channel estimation. IEEE Int. Conf. Acoust. Speech Signal Process. 3549-3552 (2012). https://doi.org/10.1109/ICASSP. 2012.6288683

28. GN Tavares, LM Tavares, The true Cramer-Rao lower bound for data-aided carrier-phase-independent time-delay estimation from linearly modulated waveforms. IEEE Trans. Commun. 54(1), 128-140 (2006). https://doi.org/10. 1109/TCOMM.2005.861655

29. S Buzzi, HV Poor, On parameter estimation in long-code DS/CDMA systems: Cramer-Rao bounds and least-squares algorithms. IEEE Trans. Signal Process. 51 (2), 545-559 (2002). https://doi.org/10.1109/TSP.2002.806987

30. C Botteron, A Host-Madsen, M Fattouche, Cramer-Rao bound for location estimation of a mobile in asynchronous DS-CDMA systems. IEEE Int. Conf. Acoust. Speech Signal Process. 4, 2221-2224 (2001). https://doi.org/10. 1109/ICASSP.2001.940439

31. C Botteron, A Host-Madsen, M Fattouche, Effects of system and environment parameters on the performance of network-based mobile station position estimators. IEEE Trans. Veh. Technol. 53(1), 163-180 (2004). https://doi.org/10.1109/TVT.2003.822029

32. K Schmeink, R Adam, PA Hoeher, Performance limits of channel parameter estimation for joint communication and positioning. EURASIP J. Adv. Signal Process. 2012, 178 (2012). https://doi.org/10.1186/1687-6180-2012-178

33. S Gezici, Z Tian, GB Giannakis, H Kobayashi, AF Molisch, HV Poor, Z Sahinoglu, Localization via ultra wideband radios: a look at positioning aspects for future sensor networks. IEEE Signal Process. Mag. 22(4), 70-84 (2005). https://doi.org/10.1109/MSP.2005.1458289

34. A Mallat, J Louveaux, L Vandendorpe, UWB based positioning in multipath channels: CRBs for AOA and for hybrid TOA-AOA based methods. IEEE Int. Conf. Commun. 5775-5780 (2007). https://doi.org/10.1109/ICC.2007.957

35. R Játiva, J Vidal, Estimacióndel Tiempo, de Llegada en un canal Rayleigh desde una perspectiva de la Cota Inferior de Cramer-Rao. Rev. Av. Cien. Ingenierías. 1(1), 5-10 (2009)

36. R Játiva, J Vidal, M Cabrera, Cramer Rao bounds in time of arrival estimation for a distributed source. IST Mob. Commun. Summit. 236-244 (2001). http://spcom.upc.edu/documents/jativa_ISTSummit2001.pdf

37. B Denis, N Daniele, NLOS Ranging error mitigation in a distributed positioning algorithm for indoor UWB Ad-Hoc Networks. Int. Workshop Wirel. Ad-Hoc Netw. 356-360 (2004). https://doi.org/10.1109/IWWAN.2004. 1525602

38. J Riba, A Urruela, A non-line-of-sight mitigation technique based on ML-detection. IEEE Int. Conf. Acoust. Speech Signal Process. 2, 153-156 (2004). https://doi.org/10.1109/ICASSP.2004.1326217

39. L Cong, W Zhuang, Nonline-of-sight error mitigation in mobile location. IEEE Trans. Wireless Commun. 4(2), 560-573 (2005). https://doi.org/10. 1109/TWC.2004.843040

40. O Yihong, $\mathrm{H}$ Kobayashi, H Suda, Analysis of wireless geolocation in a non-line-of-sight environment. IEEE Trans. Wirel. Commun. 5(3), 672-681 (2006). https://doi.org/10.1109/TWC.2006.1611097

41. KYu, YJ Guo, NLOS Error Mitigation for mobile location estimation in wireless networks. IEEE Veh. Technol. Conf, 1071-1075 (2007). https://doi. org/10.1109/NETECS.2007.228
42. JM Huerta, J Vidal, A Giremus, JY Tourneret, Joint particle filter and UKF position tracking in severe non-line-of-sight situations. IEEE J. Sel. Top. Signal Process. 3(5), 874-888 (2009). https://doi.org/10.1109/JSTSP.2009. 2027804

43. L Chen, R Piché, H Kuusniemi, R Chen, Adaptive mobile tracking in unknown non-line-of-sight conditions with application to digital TV networks. EURASIP J. Adv. Signal Process. 2014, 22 (2014). https://doi.org/ 10.1186/1687-6180-2014-22

44. C-T Chiang, P-H Tseng, K-T Feng, Hybrid TOA/TDOA based unified Kalman tracking algorithm for wireless networks. IEEE Int. Symp. Pers. Indoor Mob. Radio Commun, 1707-1712 (2010). https://doi.org/10.1109/PIMRC.2010. 5671921

45. H Li, Z Deng, Y Yu, Investigation on a NLOS Error Mitigation algorithm for TDOA Mobile Location. IET Int. Conf. Commun. Technol. Appl, 839-843 (2011). https://doi.org/10.1049/cp.2011.0787

46. Y Long, J Huang, Y Pan, J Du, Novel TOA Location algorithms based on MLE and MMSEE for NLOS environments. IEEE Int. Conf. Commun. Circ. Syst. 2, 46-49 (2013). https://doi.org/10.1109/ICCCAS.2013.6765283

47. WC Jakes, (ed.), Microwave mobile communications, 10th edn. (IEEE Press, New York, 1994)

48. TS Rappaport, Wireless Communications_principles and practice, 10th edn. (Prentice Hall, New Jersey, 1996), pp. 139-192

49. J Vidal, M Najar, M Cabrera, R Játiva, Positioning accuracy when tracking UMTS mobiles in delay and angular dispersive channels. IEEE Veh. Technol. Conf. 4, 2575-2579 (2001). https://doi.org/10.1109/VETECS.2001.944066

50. A Artés, F Pérez, J Cid, R López, C Mosquera, F Pérez, Comunicaciones Digitales, 1st edn. (Pearson Educación S.A., Madrid, 2007), pp. 470-473

51. P Laspougeas, P Pajusco, J-C Bic, Spatial radio channel for UMTS in urban small cells area. IEEE Conf. Veh. Technol. 2(885-892). https://doi.org/10. 1109/NETECF.2000.887128

52. KI Pedersen, PE Mogensen, BH Fleury, A stochastic model of the temporal and azimuthal dispersion seen at the base station in outdoor propagation environments. IEEE Trans. Veh. Technol. 49(2), 437-447 (2000). https://doi. org/10.1109/25.832975

53. LJ Greenstein, V Erceg, YS Yeh, MV Clark, A new path-gain/delay-spread propagation model for digital cellular channels. IEEE Trans. Veh. Technol. 46(2), 477-485 (1997). https://doi.org/10.1109/25.580786

54. M Nilsson, B Völcker, B Ottersten, A cluster approach to spatio-temporal channel estimation. IEEE Int. Conf. Acoust. Speech Signal Process. 5, 2757-2760 (2000). https://doi.org/10.1109/ICASSP.2000.861069

55. CD Lai, First order autoregressive Markov processes. Stoch. Process. Appl. 7(1), 65-72 (1978)

56. Y Qi, H Suda, H Kobayashi, On time-of-arrival positioning in a multipath environment. IEEE Veh. Technol. Conf. 5, 3540-3544 (2004). https://doi. org/10.1109/NETECF.2004.1404723

57. Karimi HA, (ed.), Advanced location-based technologies and services, 1st edn. (CRC Press, Boca Raton, 2013)

58. R Jativa, J Vidal, Cota Inferior de Crámer-Rao en la Estimación del Tiempo de Llegada en un canal Rice. Rev. Av. Cien. Ingenierías. 4,1, C14-C21 (2012)

59. JR Magnus, H Neudecker, Matrix differential calculus with applications in statistics and econometrics. (Wiley, New York, 1999)

60. R Raich, J Goldberg, H Messer, Bearing estimation for a distributed source: modeling, inherent accuracy limitations and algorithms. IEEE Trans. Signal Process. 48(2), 429-441 (2000). https://doi.org/10.1109/78.823970

61. C-C Chong, C-M Tan, DI Laurenson, S McLaughlin, MA Beach, AR Nix, A new statistical wideband spatio-temporal channel model for 5-GHz band WLAN systems. IEEE J. Sel. Areas Commun. 21(2), 139-150 (2003). https:// doi.org/10.1109/JSAC.2002.807347

62. J Vidal, M Nájar, R Játiva, High resolution time-of-arrival detection for wireless positioning systems. IEEE Veh. Technol. Conf. VTC-2002-Fall. 4, 2283-2287 (2002). https://doi.org/10.1109/NETECF.2002.1040627

63. X Wang, Z Wang, B O'Dea, A TOA-based location algorithm reducing the errors due to non-line-of-sight (NLOS) propagation. IEEE Trans. Veh. Technol. 52(1), 112-116 (2003). https://doi.org/10.1109/TVT.2002.807158 